\title{
Impacts of agriculture on the parasite communities of northern leopard frogs (Rana pipiens) in southern Quebec, Canada
}

\author{
K. C. KING ${ }^{1 *}$, J. D. McLAUGHLIN ${ }^{1}$, A. D. GENDRON², B. D. PAULI ${ }^{3}$, I. GIROUX ${ }^{4}$, \\ B. RONDEAU ${ }^{5}$, M. BOILY ${ }^{6}$, P. JUNEAU ${ }^{7}$ and D. J. MARCOGLIESE ${ }^{2}$ \\ ${ }^{1}$ Department of Biology, Concordia University, Montreal, Quebec, Canada H3G 1M8 \\ ${ }^{2}$ Fluvial Ecosystem Research Section, Aquatic Ecosystem Protection Research Division, Water Science and Technology \\ Directorate, Science and Technology Branch, St. Lawrence Centre, Environment Canada, 105 rue McGill, Montreal, Quebec, \\ Canada H2Y $2 E 7$ \\ ${ }^{3}$ Canadian Wildlife Service, National Wildlife Research Centre, Carleton University, Ottawa, Ontario, Canada K1A $0 H 3$ \\ ${ }^{4}$ Ministère du Développement durable, de l'Environnement et des Parcs, Direction du suivi de l'état de l'environnement, Service \\ de l'information sur les milieux aquatiques, 675 boul. René-Lévesque Est, Montreal, Quebec, Canada G1R 5V7 \\ ${ }^{5}$ Quebec Water Quality Monitoring and Surveillance Section, Water Quality Monitoring and Surveillance Science and \\ Technology Branch, Environment Canada, 105 McGill, 7 th floor, Montreal, Quebec, Canada H2Y 2E7 \\ ${ }^{6}$ Centre TOXEN, Université du Quèbec á Montréal, 141 ave. Président Kennedy, Montreal, Quebec, Canada H2X $3 Y 7$ \\ ${ }^{7}$ Department of Biological Sciences-TOXEN, Université du Quèbec à Montréal, C.P. 8888, Succ. Centre-ville, Montreal, \\ Quebec, Canada $\mathrm{H} 3 \mathrm{C} 3 \mathrm{~PB}$
}

(Received 24 Fanuary 2007; revised 8 May and 5 Fune 2007; accepted 5 Fune 2007; first published online 3 August 2007)

\section{S UMMAR Y}

Given that numerous amphibians are suffering population declines, it is becoming increasingly important to examine the relationship between disease and environmental disturbance. Indeed, while many studies relate anthropogenic activity to changes in the parasitism of snails and fishes, little is known of the impact on the parasites of amphibians, particularly from agriculture. For 2 years, the parasite communities of metamorphic northern leopard frogs from 7 agricultural wetlands were compared with those from 2 reference wetlands to study differences in parasite community diversity and abundance of various species under pristine conditions and 3 categories of disturbance: only agricultural landscape, only pesticides, and agricultural landscape with pesticides. Agricultural (and urban) area was negatively related to species richness, and associated with the near absence of adult parasites and species that infect birds or mammals. We suggest that agriculture and urbanization may hinder parasite transmission to frogs by limiting access of other vertebrate hosts of their parasites to wetlands. The only parasite found at all localities was an unidentified echinostome infecting the kidneys. This parasite dominated communities in localities surrounded by the most agricultural land, suggesting generalist parasites may persist in disrupted habitats. Community composition was associated with dissolved organic carbon and conductivity, but few links were found with pesticides. Pollution effects may be masked by a strong impact of land use on parasite transmission.

Key words: parasite communities, amphibians, species richness, Rana pipiens, agriculture, pesticides, land use, urbanization, wetlands.

\section{INTRODUCTION}

Numerous studies have examined parasitism in aquatic organisms inhabiting disturbed environments (Poulin, 1992; Lafferty, 1997; Marcogliese and Cone, 1997; Marcogliese, 2005). In fact, there is mounting experimental and field evidence that anthropogenic disturbance (e.g., urban effluents, thermal pollution, heavy metal pollution, land development) can influence the composition of parasite communities in fishes and snails (Overstreet and Howse, 1977; Khan and Thulin, 1991 ; Poulin, 1992;

* Corresponding author: Present address, Department of Biology, Indiana University, Jordan Hall 142, 1001 East Third Street, Bloomington, Indiana 47405, USA. Tel: +812855 3282.E-mail:kingkc@indiana.edu
Lafferty, 1997; MacKenzie, 1999; Lafferty and Kuris, 2005; Hernandez et al. 2007). Interestingly, the parasite communities of amphibians have rarely been examined in the context of a disturbed habitat (but see Hamann et al. 2006; Koprivnikar et al. $2006 a$; McKenzie, 2007), despite the consideration of amphibians as sentinel organisms of environmental degradation (Kiesecker et al. 2004). With populations of frogs in global decline due to various causes, including disease (Daszak et al. 2003; Kiesecker et al. 2001; Carey et al. 2003), it is worth investigating if infection levels in these animals are altered in habitats disrupted by human activity.

An increasing number of wetlands are located either near or nested within agricultural landscapes (Lemieux et al. 1995; Matson et al. 1997; Carpenter et al. 1998). Wetlands are the interface between 
terrestrial and aquatic ecosystems, and their isolation, degradation, and erosion can have drastic effects on the ecology of both environments (Gibbs, 2000; Amezaga et al. 2002), which will presumably affect the transmission of parasites within and between them. Components of agricultural runoff have been shown to increase parasite abundance in frogs by increasing susceptibility to infection through immunosuppression (Carey and Bryant, 1995 ; Kiesecker, 2002; Carey et al. 2003; Christin et al. 2003, 2004), facilitating transmission through invertebrate intermediate host availability (Johnson and Chase, 2004; McKenzie, 2007), and decreasing parasite pre-patent period (Gendron et al. 2003). Thus, parasite transmission may be enhanced in situations where immunosuppression as well as increased host abundance are overriding factors. Transmission may be reduced, however, if the parasites are directly harmed, or if contributions by hosts to the infective pool are diminished. A few pesticides, most notably atrazine, have been shown to reduce the survival and infectivity of the free-living larval stages of parasites (Pietrock and Marcogliese, 2003 ; Koprivnikar et al. 2006 b). Finally, any development of the landscape surrounding frog habitats may reduce the use of this same habitat by definitive hosts, such as birds and small carnivorous mammals, and other amphibians (Kuris and Lafferty, 1994; Huspeni and Lafferty, 2004; Hechinger and Lafferty, 2005; Koprivnikar et al. 2006a).

In this study, the helminth parasite communities of northern leopard frogs (Rana pipiens) were characterized in animals collected from wetlands exposed to varying degrees of agricultural activity in the St Lawrence River basin of southern Quebec, Canada. Populations of leopard frogs are widespread in North America where they colonize a variety of habitats, including wetlands in areas of intense agricultural activity. As metamorphs, leopard frogs are initially mainly aquatic and then become more terrestrial with age, and thus are exposed to parasites and anthropogenic disturbance in both habitats. Since species richness and diversity in parasite communities reflect those of the free-living organisms on which parasites depend for transmission and reproduction (Marcogliese and Cone, 1997; Marcogliese, 2004; Hechinger and Lafferty, 2005), a 'healthy' environment is considered to be one rich in parasite species (Marcogliese, 2005; Hudson et al. 2006). In comparison, any unhealthy or anthropogenically disturbed environment should have reduced species richness. Landscape fragmentation and wetland isolation can restrict the access of amphibians, birds, and mammals to the area, thus possibly preventing their parasites from infecting other potential hosts in that wetland habitat. If the wetlands were contaminated by agricultural pesticides, we would expect heteroxenous parasite species to be less common if aquatic invertebrate intermediate hosts and/or free-living infective stages were harmed by chemical pollution. Essentially, any physico-chemical changes to the environment that prevent hosts from occupying or using a habitat should influence the transmission and establishment of parasites, especially those that depend on trophic pathways and food web structure for infection (Cone et al. 1993; Marcogliese, 2003, 2004). Additionally, exposure to pesticides can cause immunosuppression in frogs (Gendron et al. 2003; Gilbertson et al. 2003; Christin et al. 2004), resulting in an increased abundance of certain parasites, particularly those with direct life-cycles. Consequently, the following hypotheses were examined: (1) frogs inhabiting wetlands protected from anthropogenic activity should be infected with the most parasite species, (2) frogs from wetlands surrounded by agricultural landscapes, but with otherwise uncontaminated water, will be infected with fewer parasites that use birds and mammals as definitive hosts and (3) frogs from wetlands impacted by both pesticides and landscape modifications should be infected by the fewest parasite species, although certain parasites may increase in abundance.

\section{MATERIALS AND METHODS}

\section{Study localities}

The St Lawrence River drainage basin in southern Quebec, Canada comprises tributaries and wetlands surrounded by agricultural fields largely dedicated to corn production. In brief, 7 wetlands were studied in 2004, and 2 more were added in 2005 (Fig. 1). Selection of all wetlands was based on long-term data on waterborne pesticides in rivers and water bodies in southern Quebec (Giroux, 1999, 2002) or on continuing studies related to pesticides and frogs. Two wetlands, Étang John-Sauro (Ref1) and Île Nid d'Aigle (Ref2), neither nested within agricultural land nor exposed to pesticide runoff were selected as reference localities. In addition, 7 wetlands were chosen based on 3 disturbance categories: (i) close proximity to agricultural landscape, no pesticide contamination (ii) distant from agricultural landscape, with pesticide contamination, and (iii) close proximity to agricultural landscape, with pesticide contamination. Wetlands in Parc Le Rocher (Ag1) and on Île de la Commune (Ag2) were chosen to represent the first category. Ag1 is a modified wetland within rural parkland, and although it is surrounded on a large scale by agricultural activity (and a small urban community), it is not directly adjacent to farmland and agricultural fields do not drain into it. Ag2 is adjacent to farmland, although pesticides have not been applied to the area since 2001. Rivière St François (P1) and Baie St François (P2) wetlands fit into the second category as they are not adjacent to agricultural land, yet are exposed to pesticide runoff 


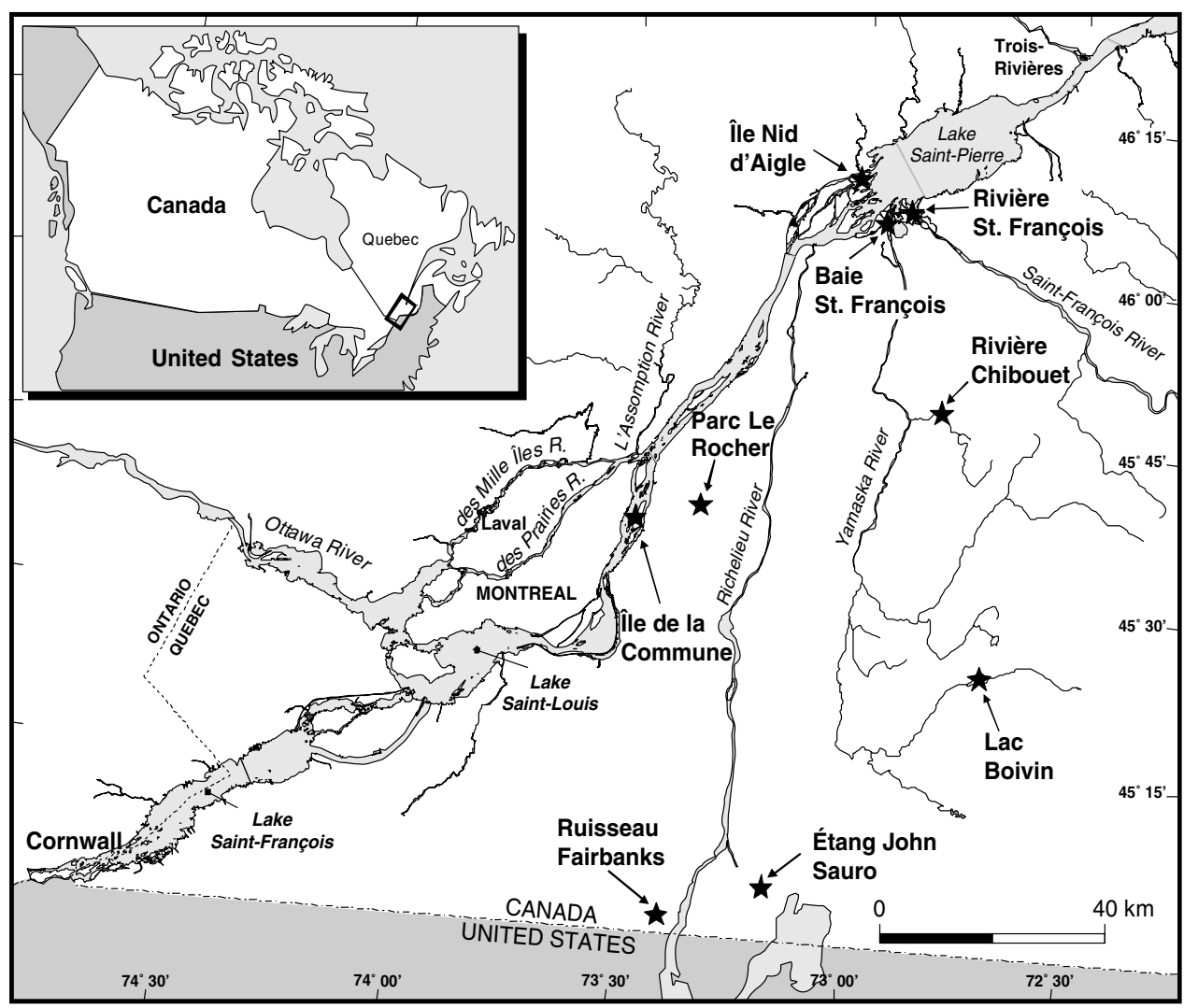

Fig. 1. Map of localities in the St Lawrence River basin in southern Quebec, Canada. Reference Localities: Étang John-Sauro (Ref1) and Île Nid d'Aigle (Ref2), agricultural landscape localities: Parc Le Rocher (Ag1) and Île de la Commune (Ag2), pesticide localities: Rivière St. François (P1) and Baie St. François (P2), agricultural landscape/ pesticide localities: Ruisseau Fairbanks (AgP1), Lac Boivin (AgP2), and Rivière Chibouet (AgP3).

through the streams and rivers draining into them. The other 3 wetlands are nested within agricultural landscapes sprayed with pesticides: Ruisseau Fairbanks (AgP1), Lac Boivin (AgP2), and Rivière Chibouet (AgP3). Despite some regulation in the water levels of Ref1 and P2, all wetlands were naturally formed, and all were verified to be leopard frog breeding habitat.

\section{Collection of environmental variables}

Water samples for pesticide and nutrient analyses were collected from May to July 2005, with the exception of pesticide samples from AgP3 that were collected in 2004 (Table 1). The timing of water collection was chosen to permit detection of peak pesticide concentrations and coincided with $R$. pipiens tadpole development. Water samples were prepared for organophosphorous pesticide and neutral herbicide analyses (Carrier, 2001). Samples from P1, P2, AgP2, and AgP3 were analysed by the Centre d'expertise en analyse environnementale du Québec of the Québec Ministère du Développement durable de l'Environnement et des Parcs (MDDEP); others were analysed at the Canadian National Laboratory for Environmental Testing in Burlington, Ontario. To measure the various nutrients (total and dissolved phosphorous, nitrates, nitrites, and dissolved organic carbon), water samples were prepared according to the Laboratoire du Centre Saint-Laurent protocol (1994). Nutrients from P1 were analysed by MDDEP, and those from the other localities were analysed by the Laboratoire des essays environnementaux du Québec (Environment Canada).

Habitat characteristics for all localities were recorded prior to and during frog collection. The surface water temperatures, surface $\mathrm{pH}$, and conductivity were recorded using a digital meter (YSI Model 63, Yellow Springs, Ohio, USA). Depth was measured at the approximate centre of the wetland. Additionally, GPS measurements were taken at points along the perimeter of the locality to permit calculation of wetland surface area. Landscape variables (forest area, urban area, agricultural area, and road area on a 100 and $500 \mathrm{~m}$ scale) were extracted using ArcGIS 9 (ESRI) from Landsat-generated satellite imagery.

Ranid tadpoles and molluscs were collected at each locality to estimate host density. Surveys were conducted in June 2005 when infected molluscs and tadpoles were expected to overlap temporally. Pipe samplers consisting of bottomless plastic garbage cans and dip nets were used to sample both molluscs and tadpoles. Sampling was stratified according to habitat type (emergent plant, submergent plant, and floating leaf) and depth range $(0-20 \mathrm{~cm}, 21-40 \mathrm{~cm}$, 
Table 1. Summary of 2005 water collections from reference and agricultural localities for pesticide and nutrient analysis

(Sampling for pesticide analysis at AgP3 was conducted in 2004. Abbreviations for localities as in Fig. 1.)

\begin{tabular}{|c|c|c|c|}
\hline \multirow[b]{2}{*}{ Locality } & \multicolumn{2}{|c|}{ Period of collection } & \multirow{2}{*}{$\begin{array}{l}\text { Sampling regime for } \\
\text { Pesticides/Nutrients }\end{array}$} \\
\hline & Pesticides & Nutrients & \\
\hline Ref1 & 23 May-4 July & 23 May-4 July & Biweekly/Biweekly \\
\hline Ref2 & 29 June & 29 June, 26 July & Once/Twice \\
\hline Ag1 & 23 May-4 July & 23 May-4 July & Biweekly/Biweekly \\
\hline $\mathrm{Ag} 2$ & 23 May-4 July & 23 May-4 July & Weekly/Biweekly \\
\hline $\mathrm{P} 1$ & 26 May-13 July & 1 June & Weekly/Once \\
\hline $\mathrm{P} 2$ & 26 May-13 July & 23 May-4 July & Weekly/Biweekly \\
\hline AgP1 & 23 May-4 July & 23 May-4 July & Weekly/Biweekly \\
\hline $\mathrm{AgP} 2$ & 8 and 28 July & 19 May-28 July & Twice/Weekly \\
\hline AgP3 & 17 May-17 July & 23 May-4 July & Triweekly/Biweekly \\
\hline
\end{tabular}

and $41-60 \mathrm{~cm})$. The sampler covered an area of $0 \cdot 1017 \mathrm{~m}^{2}$ and was randomly positioned within a stratum in the wetland. An average of 23 (15-34) samples was made at each locality, and each sample was placed at least $2 \mathrm{~m}$ from the previous one (D. Skelly, personal communication). The entire water column inside the sampler was swept from bottom to surface using dip nets (mesh size $<2 \mathrm{~mm}$ ). Ten sweeps were considered sufficient to census both tadpole and mollusc populations within the sampler (Heyer et al. 1994). Dip nets were also used for sampling along the margin. An average of 24 dip net sweeps (16-31) was taken at each wetland. In each sweep, the numbers of live snails, sphaeriid clams, and ranid tadpoles were recorded. Each was identified to family (Clarke, 1981; Desroches and Rodrigue, 2004).

\section{Host and parasite collections}

Metamorph leopard frogs were collected in both 2004 (26 July to 6 August) and 2005 (22 July to 5 August) with dip net or by hand. Only those with a snout-vent length $\leqslant 45 \mathrm{~mm}$ were kept (Seburn and Seburn, 1998). Frogs were killed in buffered $0 \cdot 8 \%$ tricaine methane sulfonate (MS222) and stored at $-80{ }^{\circ} \mathrm{C}$ until examined for parasites. The handling and treatment of animals were in accordance with the guidelines of the Canada Council on Animal Care. If the age range of the frog was in question, it was determined from the frog's longest toe phalanges (Leclair and Castanet, 1987). Frogs were weighed, measured from snout to vent, sexed, and examined for parasites according to the method of Goater and Goater (2001). Helminth parasites were identified to genus and, if possible, to species, although it was difficult to identify many to species because specimens were frozen. Identifications were based on parasite descriptions in the literature (e.g., Rau et al. 1978; Prudhoe and Bray, 1982; McAlpine and
Burt, 1998; Gillilland and Muzzall, 1999; Muzzall, 2005).

\section{Data analyses}

Quantitative descriptors were used in accordance with definitions provided by Bush et al. (1997). The parasite community structure at the various localities was examined at both the component and infracommunity levels using SPSS 13.0 and GraphPad Prism V4.0. Prevalence and mean abundance of infection were calculated for each parasite species at each locality. For non-normal data, non-parametric analyses were used or parametric analyses were performed on ranked data if more appropriate (Conover and Iman, 1981). The critical level of significance was $P<0 \cdot 05$, unless Bonferonni-corrections were applied.

Mollusc density at each locality was determined by extrapolating the number of snails and clams obtained from each sample to the number per $1 \mathrm{~m}^{2}$ of the wetland. Densities were compared by means of a Kruskal-Wallis test, followed by a Dunn's multiple comparison test.

Parasite data were screened to identify any inherent sampling biases. Mann-Whitney $U$ tests were used to test for an effect of host sex on total parasite numbers and infracommunity species richness. The snout-vent lengths (SVL) of leopard frogs were compared among localities using a KruskalWallis test. Both SVL and mass differed among localities even though all frogs were metamorphs. This variation was likely due to the plasticity of frog development and changes in ultimate size characteristics are known to result from environmental factors such as predation pressure (Laurila et al. 2002), competition (Scott, 1990), length of photo-period (Laurila et al. 2001), pesticides (Relyea, 2004), and a variety of other influences (e.g., Semlitsch et al. 1988). If SVL and the abundance of a parasite species 
Table 2. Summary of physicochemical characteristics of reference and agricultural localities in 2004

(Abbreviations for localities as in Fig. 1.)

\begin{tabular}{llllllll}
\hline \hline & Ref1 & Ag1 & Ag2 & P1 & P2 & AgP1 & AgP3 \\
\hline $\begin{array}{l}\text { Number of frogs } \\
\begin{array}{l}\text { Temperature } \\
\left({ }^{\circ} \mathrm{C}\right)\end{array}\end{array}$ & 32 & 30 & 30 & 30 & 19 & 31 & 31 \\
$\mathrm{pH}$ & $22 \cdot 9$ & $24 \cdot 1$ & $23 \cdot 9$ & $24 \cdot 0$ & - & $21 \cdot 0$ & $30 \cdot 5$ \\
$\begin{array}{l}\text { Conductivity } \\
(\mu \mathrm{S} / \mathrm{cm})\end{array}$ & $6 \cdot 72$ & $8 \cdot 50$ & $6 \cdot 72$ & $6 \cdot 72$ & - & $7 \cdot 07$ & $6 \cdot 73$ \\
$\begin{array}{l}\text { Surface area } \\
\left(\mathrm{m}^{2}\right)\end{array}$ & $123 \cdot 0$ & $199 \cdot 0$ & $175 \cdot 6$ & $124 \cdot 4$ & - & $1533 \cdot 0$ & $1430 \cdot 0$ \\
\hline \hline
\end{tabular}

were correlated at a given locality, the residuals obtained from a series of regressions of parasite species abundances with SVL were used as measures of abundance. Ranked abundances (residuals or unstandardized abundance values) were used in subsequent analyses when abundances were compared among localities. The abundance of each parasite species was compared among localities by one-way ANOVAs followed by a Tukey's post-hoc test if significant differences were detected. MANOVA was used to test if the abundance of an individual parasite species was related to the presence of pesticides at a locality (Ref1, Ref2, Ag1, Ag2 = no pesticides; P1, P2, AgP1, AgP2, AgP3 = pesticides). Univariate responses were compared using ANOVA where MANOVA results were significant. Species richness and diversity in both the infra- and component communities were determined for each locality. Species richness refers to the number of parasite species. Component community diversity was calculated using the Shannon-Wiener Diversity index, and infracommunity diversity, using Brillouin's index, at a given locality. Kruskal-Wallis tests and Dunn's multiple comparison tests were used to look for differences in infracommunity species richness and Brillouin's index values among localities. Species composition of component communities were qualitatively compared using Jaccard's similarity index (Magurran, 1988). Calculated similarity values were summarized with cluster analyses using the shareware program PHYLIP using the unweighted pair group method and arithmetic mean (UPGMA) method to give a visual interpretation of the results in the form of a dendrogram (Legendre and Legendre, 1998).

Spearman-rank correlation tests were used to evaluate associations between environmental variables measured in 2005 (snail density; nitratesnitrites; $\mathrm{P}_{\text {total }}$; dissolved organic carbon; conductivity; wetland surface area; and forest area, urban area, and agricultural area at the $500 \mathrm{~m}$ scale) and overall parasite population and community descriptors as measured by mean parasite abundance, component community species richness, mean infracommunity species richness, Shannon-Wiener diversity index, and mean Brillouin's index. Landscape variables at the $100 \mathrm{~m}$ scale were excluded because the majority of the values were zero. Road area was correlated with both agricultural and urban area at the $500 \mathrm{~m}$ scale, and so was also removed from further consideration.

Canonical correspondence analysis (CCA) was used to examine relationships among the abundance of parasite species and selected environmental characteristics of the wetlands (Legendre and Legendre, 1998) using CANOCO 4.0 (Ter Braak and Šmilauer, 1998). Although the abundance of some parasite species was associated with SVL at certain localities, there were no discernable patterns (i.e., abundance of a parasite species was not associated with SVL at all or even the majority of localities), and so host size was not considered a covariate in the analysis. In the environmental data matrix, dummy variables were used to represent the intensity of pesticide contamination (Ref1, Ref2, Ag1, Ag2=1; $\mathrm{P} 1, \mathrm{P} 2, \mathrm{AgP} 1, \mathrm{AgP} 2=2 ; \mathrm{AgP} 3=3)$. The species data matrix included the abundance of each parasite species (omitting species with an overall prevalence $\leqslant 2 \%$ ) in the 9 localities. Rare taxa were downweighted to reduce the extreme influence of rare species and of particularly high abundance values. Three environmental variables were ultimately selected from a larger set during a forward selection process whereby those variables that significantly explained variance in the parasite data were identified. These were agricultural area $(500 \mathrm{~m}$ scale), conductivity, and dissolved organic carbon. An unrestricted Monte-Carlo permutation test (with 999 permutations) was used to determine the significance of the canonical axes for parasite and locality variance.

\section{RESULTS}

\section{Habitat characteristics}

Most of the physicochemical variables varied markedly between 2004 and 2005 (Tables 2 and 3), 
Table 3. Summary of physicochemical characteristics of reference and agricultural localities sampled in 2005

(Mean values presented with ranges in parentheses. Abbreviations for localities as in Fig. 1.)

\begin{tabular}{|c|c|c|c|c|c|c|c|c|c|}
\hline & Ref1 & Ref2 & $\mathrm{Ag} 1$ & Ag2 & $\mathrm{P} 1$ & $\mathrm{P} 2$ & AgP1 & $\mathrm{AgP} 2$ & $\mathrm{AgP} 3$ \\
\hline $\begin{array}{l}\text { Number of } \\
\text { frogs }\end{array}$ & 30 & 30 & 30 & 30 & 30 & 30 & 30 & 30 & 30 \\
\hline $\begin{array}{l}\text { Temperature } \\
\left({ }^{\circ} \mathrm{C}\right)\end{array}$ & 31 & $\begin{array}{l}26 \\
(24-27)\end{array}$ & $\begin{array}{l}18 \\
(13-21)\end{array}$ & $\begin{array}{l}21 \\
(11-31)\end{array}$ & 20 & 26 & $\begin{array}{l}19 \\
(12-25)\end{array}$ & $\begin{array}{l}23 \\
(22-23)\end{array}$ & $\begin{array}{l}19 \\
(15-22)\end{array}$ \\
\hline $\mathrm{pH}$ & $7 \cdot 30$ & $\begin{array}{l}7 \cdot 5 \\
(7 \cdot 2-7 \cdot 8)\end{array}$ & $\begin{array}{l}7 \cdot 4 \\
(7 \cdot 2-8 \cdot 0)\end{array}$ & $\begin{array}{l}7 \cdot 2 \\
(6 \cdot 9-7 \cdot 5)\end{array}$ & $7 \cdot 1$ & $8 \cdot 7$ & $\begin{array}{l}7 \cdot 3 \\
(6 \cdot 9-7 \cdot 7)\end{array}$ & $\begin{array}{l}6 \cdot 8 \\
(6 \cdot 7-6 \cdot 9)\end{array}$ & $\begin{array}{l}8 \cdot 2 \\
(8 \cdot 1-8 \cdot 3)\end{array}$ \\
\hline $\begin{array}{l}\mathrm{NO}_{2}-\mathrm{NO}_{3} \\
(\mathrm{mg} / \mathrm{l})\end{array}$ & $0 \cdot 04$ & $\begin{array}{l}0 \cdot 07 \\
(0 \cdot 04-0 \cdot 1)\end{array}$ & $0 \cdot 04$ & $0 \cdot 04$ & $0 \cdot 3$ & $\begin{array}{l}1 \cdot 2 \\
(0 \cdot 04-3 \cdot 2)\end{array}$ & $\begin{array}{l}3 \cdot 3 \\
(0 \cdot 5-10 \cdot 2)\end{array}$ & $\begin{array}{l}0 \cdot 7 \\
(0 \cdot 04-3 \cdot 6)\end{array}$ & $\begin{array}{l}0 \cdot 24 \\
\quad(0 \cdot 04-0 \cdot 7)\end{array}$ \\
\hline $\begin{array}{c}\mathrm{P}_{\text {TOTAL }} \\
(\mathrm{mg} / \mathrm{l})\end{array}$ & $\begin{array}{l}0 \cdot 14 \\
\quad(0 \cdot 06-0 \cdot 25)\end{array}$ & $\begin{array}{l}0.08 \\
\quad(0 \cdot 05-0 \cdot 10)\end{array}$ & $\begin{array}{l}0 \cdot 03 \\
(0 \cdot 02-0 \cdot 04)\end{array}$ & $\begin{array}{l}0 \cdot 42 \\
\quad(0 \cdot 14-0 \cdot 94)\end{array}$ & $0 \cdot 03$ & $\begin{array}{l}0 \cdot 26 \\
(0 \cdot 13-0 \cdot 42)\end{array}$ & $\begin{array}{l}0.07 \\
\quad(0.05-0.09)\end{array}$ & $\begin{array}{l}0 \cdot 06 \\
(0 \cdot 02-0 \cdot 13)\end{array}$ & $\begin{array}{l}0 \cdot 23 \\
\quad(0 \cdot 05-0 \cdot 52)\end{array}$ \\
\hline $\begin{array}{l}\text { P PISSOLVED } \\
(\mathrm{mg} / \mathrm{l})\end{array}$ & $\begin{array}{l}0 \cdot 08 \\
(0 \cdot 05-0 \cdot 1)\end{array}$ & $\begin{array}{l}0 \cdot 05 \\
(0 \cdot 03-0 \cdot 1)\end{array}$ & $\begin{array}{l}0 \cdot 01 \\
(0 \cdot 01-0 \cdot 02)\end{array}$ & $\begin{array}{l}0 \cdot 26 \\
(0 \cdot 09-0 \cdot 7)\end{array}$ & - & $\begin{array}{l}0 \cdot 13 \\
(0 \cdot 03-0 \cdot 4)\end{array}$ & $\begin{array}{l}0 \cdot 04 \\
(0 \cdot 02-0 \cdot 05)\end{array}$ & $\begin{array}{l}0.05 \\
(0 \cdot 04-0 \cdot 06)\end{array}$ & $\begin{array}{l}0 \cdot 06 \\
(0 \cdot 02-0 \cdot 1)\end{array}$ \\
\hline $\begin{array}{l}\text { Dissolved } \\
\text { Organic }\end{array}$ & $\begin{array}{l}12 \cdot 2 \\
(8 \cdot 1-15 \cdot 8)\end{array}$ & $\begin{array}{l}8 \cdot 6 \\
(4 \cdot 7-12 \cdot 4)\end{array}$ & $\begin{array}{l}11 \cdot 6 \\
(9 \cdot 7-12 \cdot 7)\end{array}$ & $\begin{array}{l}17 \cdot 4 \\
(6 \cdot 2-27 \cdot 4)\end{array}$ & $6 \cdot 2$ & $\begin{array}{l}13 \cdot 8 \\
(8 \cdot 9-27 \cdot 2)\end{array}$ & $\begin{array}{l}7 \cdot 5 \\
(6 \cdot 0-10 \cdot 1)\end{array}$ & $\begin{array}{l}7 \cdot 7 \\
(7 \cdot 6-8 \cdot 0)\end{array}$ & $\begin{array}{l}12 \cdot 4 \\
(9 \cdot 9-14 \cdot 8)\end{array}$ \\
\hline \multicolumn{10}{|l|}{$\begin{array}{c}\text { Carbon } \\
(\mathrm{mg} / \mathrm{l})\end{array}$} \\
\hline $\begin{array}{l}\text { Conductivity } \\
(\mu \mathrm{S} / \mathrm{cm})\end{array}$ & 175 & $\begin{array}{l}182 \\
(149-207)\end{array}$ & $\begin{array}{l}309 \\
(266-381)\end{array}$ & $\begin{array}{l}777 \\
\quad(423-1130)\end{array}$ & 170 & 338 & $\begin{array}{l}427 \\
(276-595)\end{array}$ & $\begin{array}{l}168 \\
(159-177)\end{array}$ & $\begin{array}{l}643 \\
(556-730)\end{array}$ \\
\hline $\begin{array}{l}\text { Atrazine } \\
(\mu \mathrm{g} / \mathrm{l})\end{array}$ & $\begin{array}{l}0 \cdot 02 \\
\quad(0 \cdot 02-0 \cdot 04)\end{array}$ & $0 \cdot 08$ & $\begin{array}{l}0 \cdot 02 \\
(0 \cdot 01-0 \cdot 03)\end{array}$ & $\begin{array}{l}0 \cdot 04 \\
(0 \cdot 02-0 \cdot 06)\end{array}$ & $\begin{array}{l}0 \cdot 05 \\
\quad(0 \cdot 02-0 \cdot 13)\end{array}$ & $\begin{array}{l}0 \cdot 26 \\
\quad(0 \cdot 02-0 \cdot 80)\end{array}$ & $\begin{array}{l}0 \cdot 19 \\
\quad(0 \cdot 03-0 \cdot 35)\end{array}$ & $\begin{array}{l}0 \cdot 30 \\
(0 \cdot 08-0 \cdot 53)\end{array}$ & $\begin{array}{l}0 \cdot 75 \\
(0 \cdot 0-3 \cdot 70)\end{array}$ \\
\hline $\begin{array}{l}\text { Metolachlor } \\
(\mu \mathrm{g} / \mathrm{l})\end{array}$ & 0.03 & $0 \cdot 04$ & $0 \cdot 03$ & $\begin{array}{l}0 \cdot 06 \\
(0 \cdot 02-0 \cdot 15)\end{array}$ & $\begin{array}{l}0 \cdot 01 \\
\quad(0 \cdot 01-0 \cdot 03)\end{array}$ & $\begin{array}{l}0 \cdot 14 \\
\quad(0 \cdot 03-0.52)\end{array}$ & $\begin{array}{l}0 \cdot 07 \\
(0 \cdot 03-0 \cdot 14)\end{array}$ & $0 \cdot 01$ & $\begin{array}{l}0 \cdot 329 \\
(0 \cdot 04-0 \cdot 89)\end{array}$ \\
\hline $\begin{array}{l}\text { Surface area } \\
\left(\mathrm{m}^{2}\right)\end{array}$ & 3909 & 880 & 1802 & 20380 & 18270 & 6360 & 13330 & 5098 & 1453 \\
\hline $\begin{array}{l}\text { Forest* } \\
\left(\mathrm{m}^{2}\right)\end{array}$ & $1025 / 9125$ & $0 / 0$ & $0 / 7125$ & $0 / 275$ & $0 / 75$ & $0 / 1300$ & $4375 / 304375$ & $0 / 1025$ & $100 / 4525$ \\
\hline $\begin{array}{l}\text { Urban* } \\
\left(\mathrm{m}^{2}\right)\end{array}$ & $0 / 1550$ & $0 / 0$ & $125 / 2650$ & $0 / 0$ & $0 / 0$ & $0 / 525$ & $0 / 1875$ & $0 / 2700$ & $200 / 1000$ \\
\hline $\begin{array}{l}\text { Agriculture* } \\
\left(\mathrm{m}^{2}\right)\end{array}$ & $0 / 4375$ & $0 / 2500$ & $18750 / 369375$ & $1250 / 8125$ & $0 / 0$ & $0 / 0$ & $0 / 243125$ & $0 / 146875$ & $11875 / 547500$ \\
\hline $\begin{array}{l}\operatorname{Road}^{*} \\
\left(\mathrm{~m}^{2}\right)\end{array}$ & $0 / 0$ & $0 / 0$ & $0 / 26875$ & $0 / 0$ & $0 / 0$ & $625 / 10625$ & $0 / 15625$ & $0 / 39375$ & $3125 / 37500$ \\
\hline
\end{tabular}

* $100 \mathrm{~m} / 500 \mathrm{~m}$ scale. 


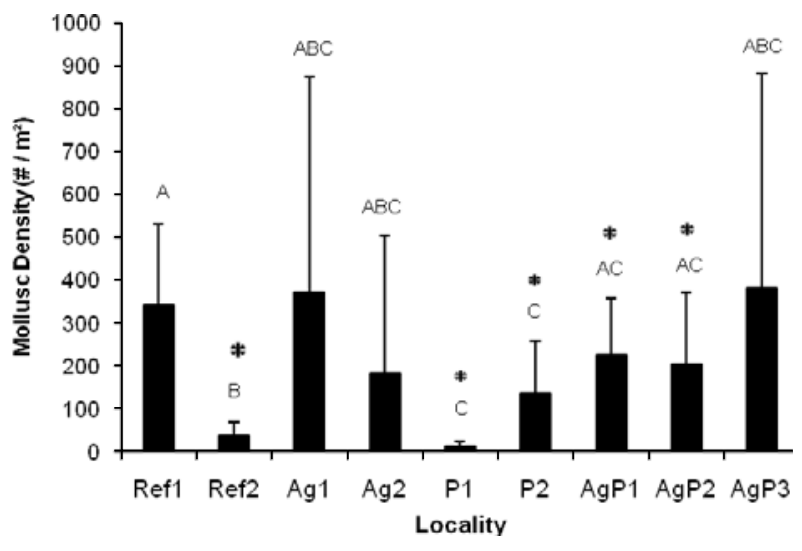

Fig. 2. Mean (+S.D.) mollusc density (no. of snails and clams $/ \mathrm{m}^{2}$ ) at reference and agricultural localities. Superscript letters indicate significant differences $(P<0 \cdot 05)$. * Localities sampled during or after heavy rainfall; other localities were sampled beforehand. Abbreviations for localities as in Fig. 1.

and did not show a consistent pattern with agricultural activity. There was great variation in nutrient levels within and among localities, but overall, the concentrations of nutrients were higher at most agricultural localities. A suite of over 30 pesticides was detected (a detailed list is available from the authors), but only concentrations of atrazine and metolachlor are presented due to their common occurrence. These 2 herbicides were found at their highest concentrations at AgP3. Atrazine levels were moderate at P1, P2, AgP1, AgP2 and only trace amounts were detected at Ref1, Ref2, Ag1, and Ag2. Except for those at AgP3, levels of metolachlor were low and relatively consistent among reference and moderately contaminated agricultural localities. The wetland surface areas of $\mathrm{Ag} 2, \mathrm{P} 1$, and $\mathrm{AgP} 1$ were the largest and those of Ref2, Ag2, and AgP3 were the smallest. At $100 \mathrm{~m}$ and $500 \mathrm{~m}$ scales, AgP1 had the greatest forest cover, Ag1 was the most urbanized, Ag1 and AgP3 were the most cultivated, and AgP3 was surrounded by the greatest road surface area.

The SVL of metamorph leopard frogs differed significantly among localities $\left(2004, \chi^{2}{ }_{6}=102 \cdot 06\right.$, $\left.P<0.001 ; 2005, \chi^{2}{ }_{8}=180.58, P<0.001\right)$, but no consistent patterns in SVL were detected within or between reference and agricultural localities from year to year. Mollusc density varied among localities $\left(\chi^{2}{ }_{8}=65.94, P<0.001\right)$ (Fig. 2). However, heavy, continuous rainfall prior to the mollusc collections at Ref2, P1, P2, AgP1, and AgP2 greatly increased water levels, and may be responsible for drastically reducing mollusc densities at these localities. Nevertheless, few consistent differences were evident between agricultural and reference localities either before or after the rainfall. Too few tadpoles were collected at each locality to accurately assess tadpole density (data not shown).
Parasite populations and communities

No sex-related differences were found in infracommunity richness $(2004, U=5105 \cdot 5, P=0.91$, $n_{\text {males }}=100, n_{\text {females }}=103 ; 2005, U=8756, P=0 \cdot 58$, $\left.n_{\text {males }}=131, n_{\text {females }}=139\right)$ or total parasite abundance $(2004, U=4505 \cdot 5, P=0 \cdot 34 ; 2005, U=9012$, $P=0 \cdot 89)$. Accordingly, data from both sexes were pooled. Eighteen helminth parasite species, including 12 digeneans and 6 nematodes, most of which used frogs as intermediate hosts, were found in 203 leopard frog metamorphs in 2004 ('Table 4). In 2005, these same species were found in 270 leopard frogs, with an additional 2 species, Halipegus sp. and Proteocephalus sp. (Table 5). Most parasites were identified to genus, but 4 groups of larval parasites were only identified to family [Echinostomatidae, Gorgoderidae, Strigeidae, and Seuratoidea (tentative identification)]. Overall, 3 larval digenean species and 1 nematode species were the most prevalent and/ or abundant parasites. In 2004, dominant species included Echinostomatidae gen. sp. 1 at Ref1, Ag2, and AgP3 infecting $94-97 \%$ of frogs at those localities; Fibricola sp. at P1 and AgP3; Gorgoderidae gen. sp. at $\mathrm{Ag} 2$; and Oswaldocruzia sp. at P1 (Table 4). In 2005, dominant species were Echinostomatidae gen. sp. 1. at Ref1, Ag1, AgP2, and P2, parasitizing $80-100 \%$ of the frogs; Fibricola sp. at Ref2, P1, and AgP3 with infection rates of over $80 \%$; and Gorgoderidae gen. sp. at Ag2 and AgP1 (Table 5). Only Echinostomatidae gen. sp. 1 was found at all localities in both years.

MANOVA was significant for both years of collection (2004, Pillai's Trace $=0 \cdot 35, P<0 \cdot 001 ; 2005$, Pillai's Trace $=0 \cdot 73, P<0 \cdot 001)$. In 2004, Fibricola sp. $(F=8 \cdot 181, P=0 \cdot 005)$ and Spiroxys sp. $(F=$ $5 \cdot 282, P=0 \cdot 023)$ were more abundant in the pesticide localities, while Apharyngostrigea pipientis ( $F=$ $9 \cdot 266, P=0 \cdot 003)$, Glypthelmins quieta $(F=8 \cdot 365$, $P=0 \cdot 005)$, Haematoloechus spp. $(F=7 \cdot 530, \quad P=$ $0 \cdot 007)$, and Rhabdias ranae $(F=13 \cdot 653, P<0 \cdot 001)$ were more abundant in those localities relatively free of pesticides. In 2005, Fibricola sp. was more abundant in pesticide localities $(F=81.598, P<0 \cdot 001)$, and only the abundance of this parasite was consistently related to pesticide contamination in both years.

The component communities at Ag1 and AgP3 were constantly among the most depauperate (7-9 species). There was little difference among the rest of the localities (12-17 species), with the exception of the component community species richness at $\mathrm{P} 2$ which was comparable to that at Ag1 and AgP3 in 2004 (8 species). Parasite component communities at Ref2, Ag1, AgP2 and AgP3 had the lowest diversity $(0.06-0.53)$ in both years, as measured by the Shannon-Wiener index. High diversity was encountered at Ref1, AgP1, and P2 in both years $(1 \cdot 25-2 \cdot 06)$, in Ag2 in $2004(1 \cdot 45)$, and in P1 in 2005 
Table 4. Prevalence (\%) and mean abundance ( $\mathrm{Ab} \pm$ s.D.) of parasite species infecting Rana pipiens in 2004

(Superscript letters indicate significant differences in abundance among localities $(P<0 \cdot 05)$. Abbreviations for localities as in Fig. 1.)

\begin{tabular}{|c|c|c|c|c|c|c|c|c|c|c|c|c|c|c|}
\hline & \multicolumn{2}{|l|}{ Ref1 } & \multicolumn{2}{|l|}{ Ag1 } & \multicolumn{2}{|l|}{$\mathrm{Ag} 2$} & \multicolumn{2}{|l|}{$\mathrm{P} 1$} & \multicolumn{2}{|l|}{$\mathrm{P} 2$} & \multicolumn{2}{|c|}{$\mathrm{AgP} 1$} & \multicolumn{2}{|c|}{$\mathrm{AgP} 3$} \\
\hline & $\%$ & $\mathrm{Ab}$ & $\%$ & $\mathrm{Ab}$ & $\%$ & $\mathrm{Ab}$ & $\%$ & $\mathrm{Ab}$ & $\%$ & $\mathrm{Ab}$ & $\%$ & $\mathrm{Ab}$ & $\%$ & $\mathrm{Ab}$ \\
\hline \multicolumn{15}{|l|}{ Digenea } \\
\hline Alaria sp.* & $3 \cdot 1$ & $0 \cdot 03^{\mathrm{B}}(0 \cdot 2)$ & & - & $16 \cdot 7$ & $0 \cdot 4^{\mathrm{AB}}(1 \cdot 2)$ & $13 \cdot 3$ & $0 \cdot 2^{\mathrm{A}}(0 \cdot 5)$ & & - & $6 \cdot 5$ & $0 \cdot 1^{\mathrm{AB}}(0 \cdot 6)$ & & - \\
\hline $\begin{array}{l}\text { Apharyngostrigea } \\
\text { pipientis } \dagger\end{array}$ & $18 \cdot 8$ & $0 \cdot 8(2 \cdot 9)$ & $3 \cdot 3$ & $0 \cdot 8(4 \cdot 2)$ & $23 \cdot 3$ & $4 \cdot 1(14 \cdot 9)$ & & - & $15 \cdot 8$ & $0 \cdot 2(0 \cdot 4)$ & $12 \cdot 9$ & $0 \cdot 7(2 \cdot 2)$ & & - \\
\hline $\begin{array}{l}\text { Clinostomum } \\
\text { sp. } \dagger\end{array}$ & $15 \cdot 6$ & $0 \cdot 3(0 \cdot 9)$ & & - & $13 \cdot 3$ & $4 \cdot 0(14 \cdot 7)$ & & - & & - & & - & & - \\
\hline $\begin{array}{l}\text { Diplostomum } \\
\text { spp. } \dagger\end{array}$ & $3 \cdot 1$ & $0 \cdot 03^{\mathrm{B}}(0 \cdot 2)$ & 10 & $0 \cdot 1^{\mathrm{B}}(0 \cdot 4)$ & $16 \cdot 7$ & $0 \cdot 2^{\mathrm{B}}(0 \cdot 6)$ & & - & & - & $32 \cdot 3$ & $0 \cdot 5^{\mathrm{A}}(0 \cdot 9)$ & & - \\
\hline $\begin{array}{l}\text { Echinostomatidae } \\
\text { gen. sp. } 1 \dagger\end{array}$ & $93 \cdot 8$ & $29 \cdot 7(36 \cdot 5)$ & $3 \cdot 3$ & $62 \cdot 6(73 \cdot 5)$ & $96 \cdot 7$ & $17 \cdot 9(19 \cdot 6)$ & $13 \cdot 3$ & $0 \cdot 6(1 \cdot 9)$ & $42 \cdot 1$ & $2 \cdot 2(4 \cdot 5)$ & $80 \cdot 7$ & $10 \cdot 8(37 \cdot 2)$ & $96 \cdot 8$ & $26 \cdot 0(53 \cdot 9)$ \\
\hline $\begin{array}{l}\text { Echinostomatidae } \\
\text { gen. sp. } 2 \dagger\end{array}$ & $31 \cdot 1$ & $0 \cdot 2(1 \cdot 1)$ & 10 & $0 \cdot 03(0 \cdot 2)$ & $3 \cdot 3$ & $0 \cdot 8(4 \cdot 6)$ & $6 \cdot 7$ & $0 \cdot 1(0 \cdot 3)$ & & - & $6 \cdot 5$ & $0 \cdot 1(0 \cdot 3)$ & & - \\
\hline Fibricola sp. $\dagger$ & $34 \cdot 4$ & $2 \cdot 2^{\mathrm{BC}}(60 \cdot 7)$ & & - & $3 \cdot 3$ & $0 \cdot 2^{\mathrm{C}}(0 \cdot 9)$ & $43 \cdot 3$ & $0 \cdot 7^{\mathrm{B}}(71 \cdot 3)$ & & - & $9 \cdot 7$ & $0 \cdot 1^{\mathrm{C}}(0 \cdot 3)$ & $80 \cdot 7$ & $175^{\mathrm{A}}(254 \cdot 9)$ \\
\hline $\begin{array}{l}\text { Glypthelmins } \\
\text { quietat }\end{array}$ & $12 \cdot 5$ & $0 \cdot 6(2 \cdot 7)$ & & - & $6 \cdot 7$ & $0 \cdot 1(0 \cdot 3)$ & $3 \cdot 3$ & $0 \cdot 03(0 \cdot 2)$ & & - & & - & & - \\
\hline $\begin{array}{c}\text { Gorgoderina } \\
\text { attenuata }\end{array}$ & $18 \cdot 8$ & $0 \cdot 2^{\mathrm{AB}}(0 \cdot 5)$ & & - & $13 \cdot 3$ & $0 \cdot 3^{\mathrm{A}}(1 \cdot 3)$ & $13 \cdot 3$ & $0 \cdot 1^{\mathrm{AB}}(0 \cdot 4)$ & & - & $35 \cdot 5$ & $0 \cdot 6^{\mathrm{B}}(0 \cdot 9)$ & & - \\
\hline $\begin{array}{l}\text { Gorgoderidae } \\
\text { gen. sp. } \dagger\end{array}$ & $21 \cdot 3$ & $5 \cdot 6(17 \cdot 6)$ & & - & $93 \cdot 3$ & $58 \cdot 3(70 \cdot 7)$ & $3 \cdot 3$ & $0 \cdot 1(0 \cdot 4)$ & $21 \cdot 6$ & $4 \cdot 1(9 \cdot 3)$ & $74 \cdot 2$ & $5 \cdot 6(6 \cdot 9)$ & $6 \cdot 5$ & $1 \cdot 1(5 \cdot 4)$ \\
\hline $\begin{array}{l}\text { Haematoloechus } \\
\text { spp.€‡. }\end{array}$ & 50 & $8 \cdot 8^{\mathrm{A}}(15 \cdot 2)$ & $3 \cdot 3$ & $0 \cdot 03^{\mathrm{B}}(0 \cdot 2)$ & $3 \cdot 3$ & $0 \cdot 2^{\mathrm{B}}(0 \cdot 9)$ & $3 \cdot 3$ & $0 \cdot 1^{\mathrm{B}}(0 \cdot 4)$ & $5 \cdot 3$ & $1 \cdot 2^{\mathrm{B}}(5 \cdot 3)$ & $35 \cdot 5$ & $5 \cdot 4^{\mathrm{A}}(16 \cdot 8)$ & & - \\
\hline $\begin{array}{l}\text { Strigeidae gen. } \\
\text { sp. } \dagger\end{array}$ & $12 \cdot 5$ & $0 \cdot 2(0 \cdot 5)$ & & - & & - & & - & $5 \cdot 3$ & $0 \cdot 1(0 \cdot 2)$ & $3 \cdot 2$ & $0 \cdot 03(0 \cdot 2)$ & $3 \cdot 2$ & $0 \cdot 1(0 \cdot 5)$ \\
\hline \multicolumn{15}{|l|}{ Nematoda } \\
\hline $\begin{array}{l}\text { Cosmocercoides } \\
\text { sp. } \neq\end{array}$ & $3 \cdot 1$ & $0 \cdot 03(0 \cdot 2)$ & & - & $3 \cdot 3$ & $1 \cdot 0(5 \cdot 5)$ & $10 \cdot 7$ & $0 \cdot 2(0 \cdot 7)$ & & - & $3 \cdot 2$ & $0 \cdot 03(0 \cdot 2)$ & & - \\
\hline $\begin{array}{l}\text { Oswaldocruzia } \\
\text { sp. } \dagger\end{array}$ & & - & $3 \cdot 3$ & $0 \cdot 03^{\mathrm{B}}(0 \cdot 18)$ & 40 & $55 \cdot 7^{\mathrm{A}}(228 \cdot 5)$ & 50 & $2 \cdot 1^{\mathrm{A}}(3 \cdot 4)$ & $15 \cdot 8$ & $1 \cdot 1^{\mathrm{AB}}(3 \cdot 1)$ & $6 \cdot 5$ & $0 \cdot 2^{\mathrm{B}}(1 \cdot 0)$ & $3 \cdot 3$ & $0 \cdot 03(0 \cdot 2)$ \\
\hline Rhabdias ranae€ & $15 \cdot 6$ & $0 \cdot 4^{\mathrm{B}}(1 \cdot 3)$ & & - & $63 \cdot 3$ & $2 \cdot 8^{\mathrm{A}}(6 \cdot 0)$ & 30 & $0 \cdot 5^{\mathrm{B}}(1 \cdot 1)$ & $21 \cdot 1$ & $1 \cdot 1^{\mathrm{B}}(3 \cdot 1)$ & $6 \cdot 5$ & $0 \cdot 2^{\mathrm{B}}(1 \cdot 0)$ & $3 \cdot 2$ & $0 \cdot 03^{\mathrm{B}}(0 \cdot 2)$ \\
\hline Spiroxys sp. $\dagger$ & $31 \cdot 3$ & $0 \cdot 4^{\mathrm{B}}(0 \cdot 6)$ & $23 \cdot 3$ & $0 \cdot 3^{\mathrm{AB}}(0 \cdot 6)$ & $6 \cdot 7$ & $0 \cdot 2^{\mathrm{AB}}(0 \cdot 8)$ & $3 \cdot 3$ & $2 \cdot 2^{\mathrm{A}}(12 \cdot 2)$ & & - & $22 \cdot 6$ & $0 \cdot 2^{\mathrm{AB}}(0 \cdot 4)$ & $3 \cdot 2$ & $0 \cdot 03^{\mathrm{B}}(0 \cdot 2)$ \\
\hline $\begin{array}{l}\text { Seuratoidea gen. } \\
\text { sp. } \dagger\end{array}$ & 50 & $5 \cdot 7^{\mathrm{A}}(9 \cdot 2)$ & & - & $3 \cdot 3$ & $0 \cdot 2^{\mathrm{C}}(0 \cdot 9)$ & $3 \cdot 3$ & $1 \cdot 9^{\mathrm{C}}(10 \cdot 2)$ & & - & $90 \cdot 3$ & $4 \cdot 1^{\mathrm{B}}(3 \cdot 4)$ & $3 \cdot 2$ & $0.03^{\mathrm{C}}(0 \cdot 2)$ \\
\hline $\begin{array}{l}\text { Strongyloides } \\
\text { sp.t }\end{array}$ & $3 \cdot 1$ & $31 \cdot 2(176 \cdot 6)$ & & - & $16 \cdot 7$ & $1 \cdot 5(5 \cdot 5)$ & $23 \cdot 3$ & $0 \cdot 7(1 \cdot 7)$ & $5 \cdot 3$ & $0 \cdot 3(1 \cdot 1)$ & $22 \cdot 6$ & $33 \cdot 5(179 \cdot 2)$ & $3 \cdot 2$ & $0 \cdot 03(0 \cdot 2)$ \\
\hline
\end{tabular}

* Mesocercaria.

$€$ Immature.

$\dagger$ Larva/Metacercaria.

* Adult. 
Table 5. Prevalence (\%) and mean abundance ( $\mathrm{Ab} \pm$ s.D.) of parasite species infecting Rana pipiens in 2005

(Superscript letters indicate significant differences in abundance among localities $(P<0 \cdot 05)$. Abbreviations for localities as in Fig. 1.)

\begin{tabular}{|c|c|c|c|c|c|c|c|c|c|c|c|c|c|c|c|c|c|c|}
\hline \multirow[b]{2}{*}{ Parasite Species } & \multicolumn{2}{|l|}{ Ref1 } & \multicolumn{2}{|l|}{ Ref2 } & \multicolumn{2}{|l|}{ Ag1 } & \multicolumn{2}{|l|}{$\mathrm{Ag} 2$} & \multicolumn{2}{|l|}{$\mathrm{P} 1$} & \multicolumn{2}{|l|}{$\mathrm{P} 2$} & \multicolumn{2}{|l|}{$\mathrm{AgP} 1$} & \multicolumn{2}{|c|}{$\mathrm{AgP} 2$} & \multicolumn{2}{|c|}{$\mathrm{AgP} 3$} \\
\hline & $\%$ & $\mathrm{Ab}$ & $\%$ & $\mathrm{Ab}$ & $\%$ & $\mathrm{Ab}$ & $\%$ & $\mathrm{Ab}$ & $\%$ & $\mathrm{Ab}$ & $\%$ & $\mathrm{Ab}$ & $\%$ & $\mathrm{Ab}$ & $\%$ & $\mathrm{Ab}$ & $\%$ & $\mathrm{Ab}$ \\
\hline \multicolumn{19}{|l|}{ Digenea } \\
\hline Alaria sp.* & 30 & $\begin{array}{l}1 \cdot 3 \\
(2 \cdot 9)\end{array}$ & $13 \cdot 3$ & $\begin{array}{l}1 \cdot 0 \\
(4 \cdot 9)\end{array}$ & - & & $26 \cdot 7$ & $\begin{array}{l}0 \cdot 3 \\
(0 \cdot 5)\end{array}$ & $13 \cdot 3$ & $\begin{array}{l}0 \cdot 4 \\
(1 \cdot 5)\end{array}$ & 30 & $\begin{array}{l}1 \cdot 8 \\
(4 \cdot 7)\end{array}$ & 10 & $\begin{array}{l}0 \cdot 3 \\
(1 \cdot 0)\end{array}$ & $13 \cdot 3$ & $\begin{array}{l}0 \cdot 9 \\
(4 \cdot 6)\end{array}$ & $3 \cdot 3$ & $\begin{array}{l}0 \cdot 1 \\
(0 \cdot 7)\end{array}$ \\
\hline $\begin{array}{l}\text { Apharyngostrigea } \\
\text { pipientis } \dagger\end{array}$ & 10 & $\begin{array}{l}0 \cdot 1 \\
(0 \cdot 4)\end{array}$ & $13 \cdot 3$ & $\begin{array}{l}0 \cdot 1 \\
(0 \cdot 4)\end{array}$ & 10 & $\begin{array}{l}0 \cdot 5 \\
(1 \cdot 6)\end{array}$ & $13 \cdot 3$ & $\begin{array}{l}0 \cdot 2 \\
(0 \cdot 5)\end{array}$ & 10 & $\begin{array}{l}0 \cdot 1 \\
(0 \cdot 3)\end{array}$ & $63 \cdot 3$ & $\begin{array}{l}2 \cdot 8 \\
(4 \cdot 4)\end{array}$ & $16 \cdot 7$ & $\begin{array}{l}0 \cdot 3 \\
(0 \cdot 8)\end{array}$ & $26 \cdot 7$ & $\begin{array}{c}1 \cdot 6 \\
(4 \cdot 1)\end{array}$ & & - \\
\hline Clinostomum sp. $\dagger$ & $16 \cdot 7$ & $\begin{array}{l}0 \cdot 4 \\
(1 \cdot 3)\end{array}$ & & - & & - & & - & & - & & - & & - & $6 \cdot 7$ & $\begin{array}{l}0 \cdot 3 \\
(1 \cdot 5)\end{array}$ & & - \\
\hline Diplostomum spp $\uparrow$ & & - & $83 \cdot 3$ & $\begin{array}{l}19 \cdot 9 \\
(23 \cdot 9)\end{array}$ & $33 \cdot 3$ & $\begin{array}{l}2 \cdot 0 \\
(6 \cdot 9)\end{array}$ & $13 \cdot 3$ & $\begin{array}{l}0 \cdot 1 \\
(0 \cdot 4)\end{array}$ & $6 \cdot 7$ & $\begin{array}{l}0 \cdot 1 \\
(0 \cdot 3)\end{array}$ & & - & $33 \cdot 3$ & $\begin{array}{l}1 \cdot 2 \\
(5 \cdot 5)\end{array}$ & & - & & - \\
\hline $\begin{array}{l}\text { Echinostomatidae } \\
\text { gen. sp. } 1 \dagger\end{array}$ & 100 & $\begin{array}{l}44 \cdot 8 \\
(53 \cdot 9)\end{array}$ & 70 & $\begin{array}{l}13 \cdot 2 \\
(28 \cdot 6)\end{array}$ & 100 & $\begin{array}{l}52 \cdot 8 \\
(206 \cdot 9)\end{array}$ & $93 \cdot 3$ & $\begin{array}{c}10 \cdot 2 \\
(9 \cdot 3)\end{array}$ & 60 & $\begin{array}{l}9 \cdot 8 \\
(33 \cdot 2)\end{array}$ & $93 \cdot 8$ & $\begin{array}{l}8 \cdot 1 \\
(12 \cdot 1)\end{array}$ & $46 \cdot 7$ & $\begin{array}{l}2 \cdot 7 \\
(4 \cdot 6)\end{array}$ & 80 & $\begin{array}{l}42 \cdot 6 \\
(75 \cdot 0)\end{array}$ & $56 \cdot 7$ & $\begin{array}{l}5 \cdot 6 \\
(9 \cdot 9)\end{array}$ \\
\hline $\begin{array}{l}\text { Echinostomatidae } \\
\text { gen. sp. } 2 \dagger\end{array}$ & & - & $3 \cdot 3$ & $\begin{array}{l}0 \cdot 03 \\
(0 \cdot 2)\end{array}$ & & - & & - & $3 \cdot 3$ & $\begin{array}{l}0 \cdot 03 \\
(0 \cdot 2)\end{array}$ & & - & 6.7 & $\begin{array}{l}0 \cdot 4 \\
(1 \cdot 9)\end{array}$ & & - & & - \\
\hline Fibricola sp. $\dagger$ & 70 & $\begin{array}{l}20 \cdot 4^{\mathrm{B}} \\
(49 \cdot 3)\end{array}$ & 90 & $\begin{array}{l}348^{A} \\
(525 \cdot 3)\end{array}$ & $6 \cdot 7$ & $\begin{array}{l}0 \cdot 1^{\mathrm{B}} \\
(0 \cdot 4)\end{array}$ & $83 \cdot 3$ & $\begin{array}{l}6 \cdot 1^{\mathrm{B}} \\
(5 \cdot 7)\end{array}$ & 100 & $\begin{array}{l}2374^{A} \\
(1354)\end{array}$ & $43 \cdot 3$ & $\begin{array}{l}38^{\mathrm{B}} \\
(185 \cdot 8)\end{array}$ & $23 \cdot 3$ & $\begin{array}{l}3 \cdot 5^{\mathrm{C}} \\
(11 \cdot 3)\end{array}$ & $23 \cdot 3$ & $\begin{array}{c}14^{\mathrm{B}} \\
(46)\end{array}$ & 80 & $\begin{array}{c}33 \cdot 1^{\mathrm{B}} \\
(46)\end{array}$ \\
\hline $\begin{array}{l}\text { Glypthelmins } \\
\text { quieta: }\end{array}$ & $26 \cdot 7$ & $\begin{array}{c}1 \cdot 1^{\mathrm{A}} \\
(2 \cdot 6)\end{array}$ & & - & & - & $3 \cdot 3$ & $\begin{array}{r}0 \cdot 03^{\mathrm{B}} \\
(0 \cdot 2)\end{array}$ & $3 \cdot 3$ & $\begin{array}{c}0 \cdot 1^{\mathrm{B}} \\
(0 \cdot 4)\end{array}$ & $3 \cdot 3$ & $\begin{array}{c}0 \cdot 03^{\mathrm{B}} \\
(0 \cdot 2)\end{array}$ & $6 \cdot 7$ & $\begin{array}{l}0 \cdot 1^{\mathrm{B}} \\
(0 \cdot 3)\end{array}$ & 10 & $\begin{array}{c}0 \cdot 2^{\mathrm{B}} \\
(0 \cdot 6)\end{array}$ & & - \\
\hline $\begin{array}{l}\text { Gorgoderina } \\
\text { attenuata: }\end{array}$ & 10 & $\begin{array}{r}0 \cdot 1^{\mathrm{AB}} \\
(0 \cdot 4)\end{array}$ & $36 \cdot 7$ & $\begin{array}{l}0 \cdot 6^{\mathrm{A}} \\
(0 \cdot 9)\end{array}$ & & - & 57 & $\begin{array}{r}1 \cdot 1^{\mathrm{AB}} \\
(1 \cdot 6)\end{array}$ & $23 \cdot 3$ & $\begin{array}{c}0 \cdot 3^{\mathrm{B}} \\
(0 \cdot 7)\end{array}$ & $3 \cdot 3$ & $\begin{array}{c}0 \cdot 03^{A B} \\
(0 \cdot 2)\end{array}$ & & $\begin{array}{r}0 \cdot 2^{\mathrm{AB}} \\
(0 \cdot 6)\end{array}$ & $3 \cdot 3$ & $\begin{array}{r}0 \cdot 1^{\mathrm{AB}} \\
(0 \cdot 4)\end{array}$ & & - \\
\hline $\begin{array}{l}\text { Gorgoderidae } \\
\text { gen. sp. } \dagger\end{array}$ & 87 & $\begin{array}{r}43 \cdot 4 \\
(86 \cdot 9)\end{array}$ & 40 & $\begin{array}{l}0 \cdot 9 \\
(1 \cdot 5)\end{array}$ & $6 \cdot 7$ & $\begin{array}{l}0 \cdot 4 \\
(1 \cdot 5)\end{array}$ & 100 & $\begin{array}{l}128 \\
(59 \cdot 2)\end{array}$ & $33 \cdot 3$ & $\begin{array}{l}0 \cdot 9 \\
(1.9)\end{array}$ & $93 \cdot 3$ & $\begin{array}{l}33 \cdot 5 \\
(45 \cdot 2)\end{array}$ & 50 & $\begin{array}{c}1 \cdot 8 \\
(3 \cdot 1)\end{array}$ & $46 \cdot 7$ & $\begin{array}{l}9 \cdot 4 \\
(35 \cdot 1)\end{array}$ & & - \\
\hline $\begin{array}{l}\text { Haematoloechus } \\
\text { spp.€‡. }\end{array}$ & 50 & $\begin{array}{c}12 \cdot 2^{\mathrm{A}} \\
(21 \cdot 6)\end{array}$ & $6 \cdot 7$ & $\begin{array}{l}0 \cdot 1^{\mathrm{B}} \\
(0 \cdot 3)\end{array}$ & $6 \cdot 7$ & $\begin{array}{r}0 \cdot 2^{\mathrm{B}} \\
(0 \cdot 6)\end{array}$ & $3 \cdot 3$ & $\begin{array}{c}0 \cdot 03^{\mathrm{B}} \\
(0 \cdot 2)\end{array}$ & $3 \cdot 3$ & $\begin{array}{c}1 \cdot 5^{\mathrm{B}} \\
(8 \cdot 2)\end{array}$ & & - & $3 \cdot 3$ & $\begin{array}{l}0 \cdot 1^{\mathrm{B}} \\
(0 \cdot 7)\end{array}$ & $3 \cdot 3$ & $\begin{array}{l}0 \cdot 1^{\mathrm{B}} \\
(0 \cdot 6)\end{array}$ & & - \\
\hline Halipegus sp.€ & & - & & - & & - & & - & & - & & - & - & & & - & $3 \cdot 3$ & $\begin{array}{c}0 \cdot 03 \\
(0 \cdot 2)\end{array}$ \\
\hline $\begin{array}{l}\text { Strigeidae } \\
\text { gen. sp. } \dagger\end{array}$ & & - & & - & & - & & - & & - & & - & $3 \cdot 3$ & $\begin{array}{l}0 \cdot 1 \\
(0 \cdot 7)\end{array}$ & $3 \cdot 3$ & $\begin{array}{l}0 \cdot 1 \\
(0 \cdot 4)\end{array}$ & & - \\
\hline \multicolumn{19}{|l|}{ Nematoda } \\
\hline Cosmocercoides sp $\$$ & $6 \cdot 7$ & $\begin{array}{l}0 \cdot 1 \\
(0 \cdot 6)\end{array}$ & $3 \cdot 3$ & $\begin{array}{l}0 \cdot 1 \\
(0 \cdot 7)\end{array}$ & 10 & $\begin{array}{l}0 \cdot 2 \\
(0 \cdot 8)\end{array}$ & 6.7 & $\begin{array}{l}1 \cdot 0 \\
(4 \cdot 0)\end{array}$ & $36 \cdot 7$ & $\begin{array}{l}1 \cdot 1 \\
(3 \cdot 1)\end{array}$ & & - & 10 & $\begin{array}{l}0 \cdot 6 \\
(2 \cdot 6)\end{array}$ & $36 \cdot 7$ & $\begin{array}{l}1 \cdot 3 \\
(2 \cdot 7)\end{array}$ & & - \\
\hline Oswaldocruzia sp $甘$ & $6 \cdot 7$ & $\begin{array}{c}0 \cdot 1^{\mathrm{A}} \\
(0 \cdot 4)\end{array}$ & 33 & $\begin{array}{r}3 \cdot 9^{\mathrm{AB}} \\
(8 \cdot 2)\end{array}$ & $3 \cdot 3$ & $\begin{array}{c}0 \cdot 03^{\mathrm{AB}} \\
(0 \cdot 2)\end{array}$ & 10 & $\begin{array}{r}0 \cdot 1^{\mathrm{AB}} \\
(0 \cdot 4)\end{array}$ & $6 \cdot 7$ & $\begin{array}{r}1 \cdot 4^{\mathrm{AB}} \\
(4 \cdot 0)\end{array}$ & 20 & $\begin{array}{r}0 \cdot 2^{\mathrm{AB}} \\
(0 \cdot 5)\end{array}$ & $6 \cdot 7$ & $\begin{array}{l}0 \cdot 4^{\mathrm{B}} \\
(1 \cdot 5)\end{array}$ & $13 \cdot 3$ & $\begin{array}{c}0 \cdot 3^{\mathrm{B}} \\
(1 \cdot 0)\end{array}$ & $3 \cdot 3$ & $\begin{array}{c}0 \cdot 03^{\mathrm{AB}} \\
(0 \cdot 2)\end{array}$ \\
\hline Rhabdias ranae: & $13 \cdot 3$ & $\begin{array}{l}0 \cdot 2 \\
(0 \cdot 6)\end{array}$ & 70 & $\begin{array}{l}4 \cdot 5 \\
(7 \cdot 1)\end{array}$ & $16 \cdot 7$ & $\begin{array}{l}0 \cdot 2 \\
(0 \cdot 5)\end{array}$ & 33 & $\begin{array}{l}0 \cdot 5 \\
(1 \cdot 0)\end{array}$ & $23 \cdot 3$ & $\begin{array}{l}0 \cdot 5 \\
(1 \cdot 0)\end{array}$ & $26 \cdot 7$ & $\begin{array}{l}0 \cdot 6 \\
(1 \cdot 4)\end{array}$ & - & & $16 \cdot 7$ & $\begin{array}{l}0 \cdot 6 \\
(1 \cdot 9)\end{array}$ & $3 \cdot 3$ & $\begin{array}{c}0 \cdot 03 \\
(0 \cdot 2)\end{array}$ \\
\hline Spiroxys sp. $\dagger$ & $16 \cdot 7$ & $\begin{array}{l}0 \cdot 2 \\
(0 \cdot 6)\end{array}$ & $3 \cdot 3$ & $\begin{array}{c}0 \cdot 03 \\
(0 \cdot 2)\end{array}$ & & - & $6 \cdot 7$ & $\begin{array}{l}0 \cdot 1 \\
(0 \cdot 3)\end{array}$ & $6 \cdot 7$ & $\begin{array}{l}0 \cdot 1 \\
(0 \cdot 4)\end{array}$ & 10 & $\begin{array}{l}0 \cdot 1 \\
(0 \cdot 3)\end{array}$ & $3 \cdot 3$ & $\begin{array}{l}0 \cdot 03 \\
(0 \cdot 2)\end{array}$ & $3 \cdot 3$ & $\begin{array}{l}0 \cdot 1 \\
(0 \cdot 4)\end{array}$ & & - \\
\hline Seuratoidea gen. sp. $\dagger$ & 33 & $\begin{array}{r}1 \cdot 4^{\mathrm{AB}} \\
(2 \cdot 9)\end{array}$ & & - & & - & & - & & - & $3 \cdot 3$ & $\begin{array}{r}0 \cdot 03^{\mathrm{B}} \\
(0 \cdot 2)\end{array}$ & - & & $23 \cdot 3$ & $\begin{array}{c}0 \cdot 4^{\mathrm{A}} \\
(0 \cdot 8)\end{array}$ & & - \\
\hline Strongyloides sp.\$ & $13 \cdot 3$ & $\begin{array}{c}1 \cdot 3 \\
(5 \cdot 5)\end{array}$ & $16 \cdot 7$ & $\begin{array}{l}0 \cdot 5 \\
(1 \cdot 4)\end{array}$ & & - & 50 & $\begin{array}{l}6 \cdot 4 \\
(9 \cdot 3)\end{array}$ & 60 & $\begin{array}{l}2 \cdot 6 \\
(3 \cdot 8)\end{array}$ & 10 & $\begin{array}{l}0 \cdot 3 \\
(1 \cdot 2)\end{array}$ & - & & $26 \cdot 7$ & $\begin{array}{l}2 \cdot 3 \\
(7 \cdot 9)\end{array}$ & $3 \cdot 3$ & $\begin{array}{c}0 \cdot 03 \\
(0 \cdot 2)\end{array}$ \\
\hline \multicolumn{19}{|l|}{ Cestoda } \\
\hline Proteocephalus sp. $\dagger$ & & - & $3 \cdot 3$ & $\begin{array}{c}0 \cdot 03 \\
(0 \cdot 2)\end{array}$ & & - & & - & - & & - & & 6.7 & $\begin{array}{l}0 \cdot 3 \\
(1 \cdot 2)\end{array}$ & & - & & - \\
\hline
\end{tabular}

* Mesocercaria

$€$ Immature

Larva/Metacercaria. 


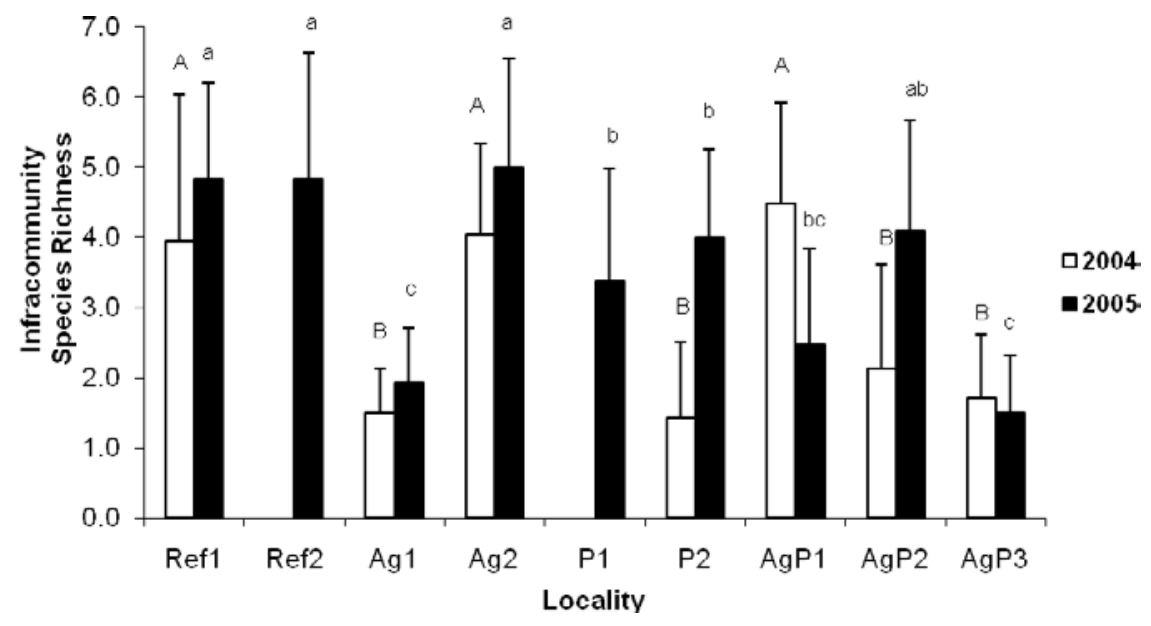

Fig. 3. Mean (+S.D.) parasite infracommunity species richness of Rana pipiens at reference and agricultural localities in 2004 and 2005. Superscript letters indicate significant differences $(P<0 \cdot 05)$ among localities within a year (upper case: 2004, lower case: 2005). Abbreviations for localities as in Fig. 1.

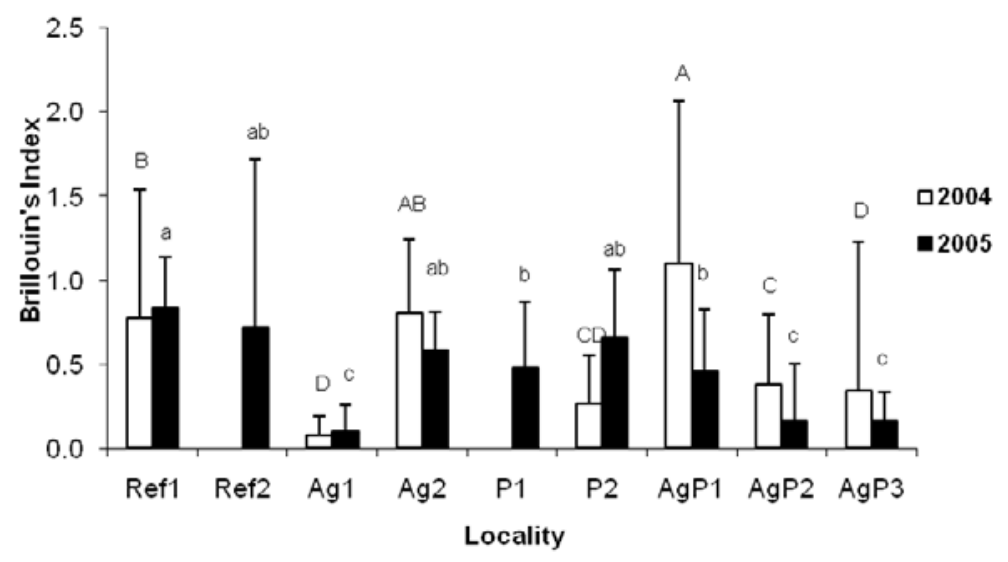

Fig. 4. Mean (+S.D.) Brillouin's Diversity Index for parasite infracommunities of Rana pipiens at reference and agricultural localities in 2004 and 2005. Superscript letters indicate significant differences $(P<0$-05) among localities within a year (upper case: 2004, lower case: 2005). Abbreviations for localities as in Fig. 1.

$(1 \cdot 36)$. Differences in infracommunity species richness varied significantly among localities (2004, $\left.\chi^{2}{ }_{6}=102 \cdot 25, P<0 \cdot 001 ; 2005, \chi^{2}{ }_{8}=134 \cdot 35, P<0 \cdot 001\right)$ (Fig. 3). In 2004, frogs at Ref1, Ag2 and AgP1 harboured the most species-rich parasite infracommunities, while those from Ag1, P1, P2, AgP2 and $\mathrm{AgP} 3$ had the least number of species. In 2005, species richness in frogs from Ag1 and AgP3 were significantly lower than those from all other localities. Brillouin's diversity index for parasite infracommunities corroborated the general pattern seen at the component community level (Fig. 4). Significant differences were observed among localities in 2004 and $2005\left(2004, \chi^{2}{ }_{6}=91 \cdot 93, P<0 \cdot 001\right.$; $\left.2005, \chi^{2}{ }_{8}=107 \cdot 37, P<0 \cdot 001\right)$. In 2004 , parasite infracommunities of frogs at Ref1, Ag2, and AgP1 were the most diverse, while those at Ag1, P2, and AgP3 were the least diverse. In 2005, Ag1, P1, and AgP3 consistently had the lowest infracommunity diversities relative to the other localities. Values for
Jaccard's index for component communities ranged from 0.21 to 0.82 in 2004 and 0.22 to 0.93 in 2005 . The dendrograms based on Jaccard's similarity in 2004 (Fig. 5A) and 2005 (Fig. 5B) reinforce the differences in species composition between Ag1 and AgP3 and the communities from other wetlands. In addition to P2 in 2004, both Ag1 and AgP3 were clearly separated from the other localities based on parasite species occurrence, and their component communities were the most dissimilar from the others in 2004 and 2005, as well as being very dissimilar from each other.

\section{Environment-community analyses}

Mean infracommunity species richness was negatively correlated with urban area (Spearman's Rho $=-0.86, \quad P=0.01)$ and agricultural area (Rho $=-0.69, P=0.04)$ at the $500 \mathrm{~m}$ scale. Mean total parasite abundance was negatively correlated 

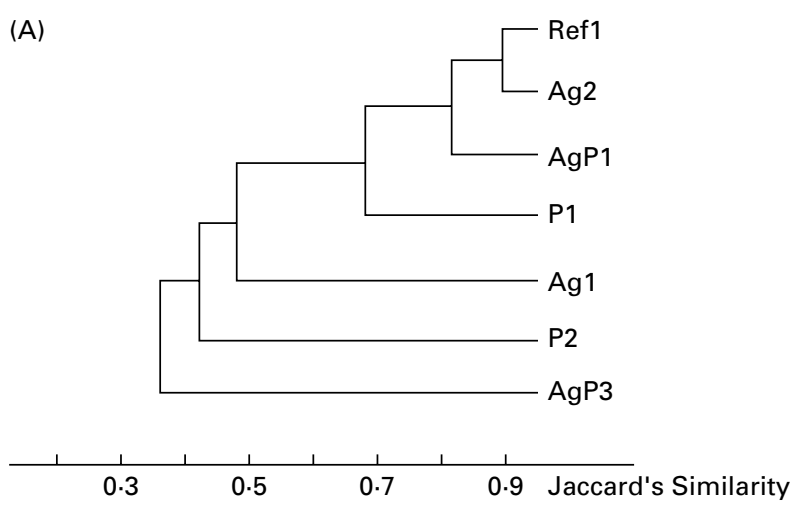

(B)

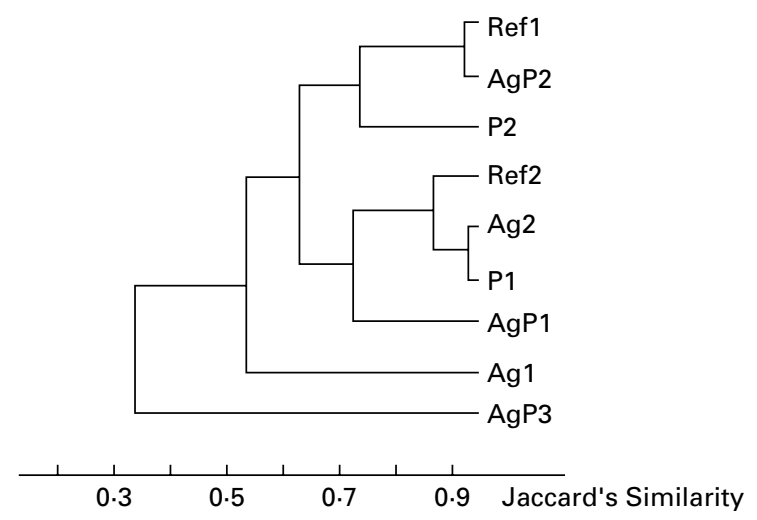

Fig. 5. Dendrogram from UPGMA cluster analysis on parasite communities of Rana pipiens in reference and agricultural localities (A) in 2004, and (B) in 2005. The clusters are based on Jaccard's similarity indices of the species composition of parasite component communities. Abbreviations for localities as in Fig. 1.

with nitrates-nitrites $(R h o=-0 \cdot 77, P=0 \cdot 01)$ and forest area surrounding the localities $(R h o=-0 \cdot 78$, $P=0 \cdot 01)$. Component community species richness was negatively correlated with dissolved organic carbon (Rho $=-0 \cdot 73, P=0 \cdot 03)$. Parasite communities and localities were distinguished further using CCA. The CCA model was significant for the first canonical axis $(F=3 \cdot 376, P=0 \cdot 040)$ and for all 4 axes $(F=5 \cdot 730, P=0 \cdot 015)$. Axis 1 explained $57 \cdot 0 \%$ and Axis 2 accounted for a further $43.0 \%$ of the variation in the abundance of parasite species versus environment relationships. The sum of all unconstrained eigenvalues was 1.586 and that of all canonical eigenvalues was $1 \cdot 229$. Axis 1 had the larger eigenvalue $(0.639)$ compared with the eigenvalue of Axis $2(0.547)$ and therefore, the former axis defined the clearest gradient. The correlation coefficients showed that Axis 1 was most strongly associated with increasing dissolved organic carbon $(r=0.75)$, whereas Axis 2 represented a gradient of decreasing agricultural area $(r=-0.92)$ and increasing conductivity $(r=0 \cdot 83)$. On the ordination, the abundance of most parasite species was positively associated with dissolved organic carbon and conductivity and negatively associated with agricultural
(A)

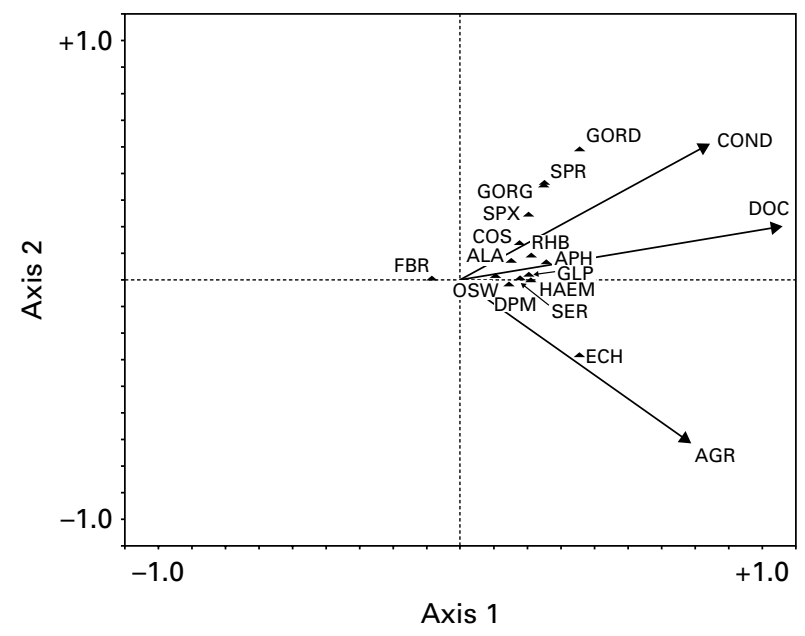

(B)

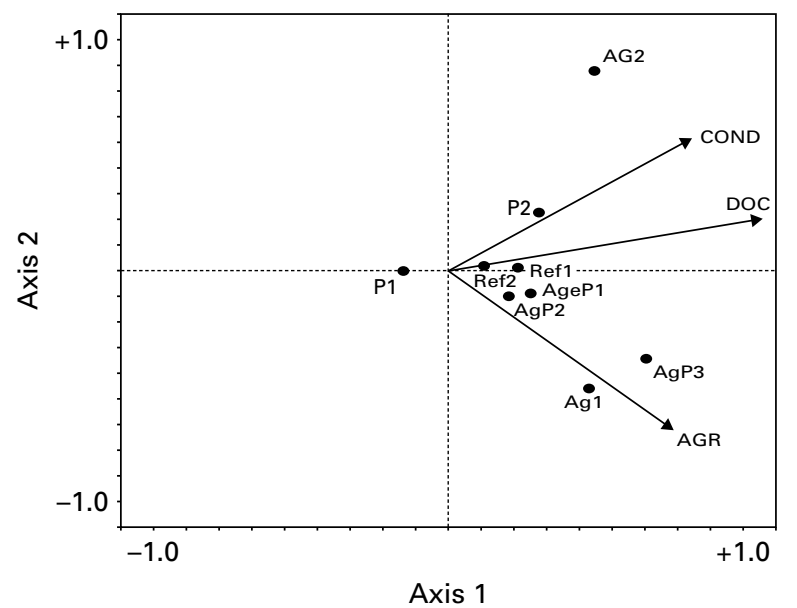

Fig. 6 (A) CCA biplot of environmental variables and parasite species abundance in Rana pipiens from reference and agricultural localities. Parasite species abbreviations: $\mathrm{ALA}=$ Alaria $\mathrm{sp}$;

$\mathrm{APH}=$ Apharyngostrigea pipientis $; \mathrm{COS}=$ Cosmocercoides sp.; DPM = Diplostomum spp.; ECH = Echinostomatidae gen. sp. 1; FIB = Fibricola $\mathrm{sp}$; $\mathrm{GLP}=$ Glypthelmins quieta; GORD = Gorgoderidae gen. sp.;

GORG $=$ Gorgoderina attenuata;

$\mathrm{HAEM}=$ Haematoloechus $\mathrm{spp}$; $\mathrm{OSW}=$ Oswaldocruzia $\mathrm{sp}$. $\mathrm{RHB}=$ Rhabdias ranae $; \mathrm{SER}=$ Seuratoidea gen. $\mathrm{sp}$. $\mathrm{SPX}=$ Spiroxys $\mathrm{sp} . ; \mathrm{SRG}=$ Strongyloides $\mathrm{sp}$.

Environmental variable abbreviations:

AGR $=$ Agricultural area COND $=$ Conductivity; $\mathrm{DOC}=$ Dissolved organic carbon. (B) CCA biplot of environmental variables and localities.

area (Fig. 6A), however, with 2 notable exceptions. Firstly, the abundance of Fibricola sp. was negatively related to dissolved organic carbon, but not to conductivity or agricultural area. Secondly, abundance of Echinostomatidae gen. sp. 1 was positively associated with agricultural area. Abundance of both gorgoderids (metacercariae and adults) and Strongyloides sp. were strongly and positively 
associated with Axis 1, and thus dissolved organic carbon. Wetlands surrounded by large areas of agricultural land formed the most distinct group on the ordination (Fig. 6B), regardless of whether they were contaminated with pesticides. Ag1, AgP1, $\mathrm{AgP} 2$, and $\mathrm{AgP} 3$ were all positively associated with agricultural area, with $\mathrm{Ag} 1$ and $\mathrm{AgP} 3$ demonstrating the strongest relationship. P2 and Ag2 were positively related to Axis 2, associated with the high levels of conductivity recorded at the 2 localities. With the exception of $\mathrm{P} 1$, all of the localities were positively associated with Axis 1 . The parasite communities of frogs from wetlands surrounded by the largest areas of agricultural land were dominated by Echinostomatidae gen. sp. 1 (Ag1, AgP3), while those from P1 were dominated by Fibricola sp. Additionally, Ref1 and Ref2 had many co-occurring parasites, including Haematoloechus spp., Glypthelmins quieta, Seuratoidea gen. sp., Cosmocercoides sp., Oswaldocruzia sp., and Rhabdias ranae. Strongyloides sp. and gorgoderids were also encountered in high abundance at P2 and Ag2, localities with high conductivity.

\section{DISCUSSION}

The parasites of leopard frogs appear to have responded to environmental disturbance from agricultural activity, and specifically from landscape modification. While there were few differences between the parasite communities of the protected wetlands and 5 of the agricultural localities, consistently low species richness and diversity were found at 2 wetlands, Ag1 and AgP3. These were surrounded by the most agricultural (and generally developed) land, with only the latter being highly pesticide contaminated. These patterns in parasite communities are consistent with many previous studies of parasitism in substantially disturbed environments. Decreases in species richness and diversity in the helminth parasite communities of fishes and snails can indeed result from aquatic pollution or development in the surrounding landscape (Lafferty, 1997; Marcogliese, 2004, 2005). As predicted in localities with landscape disturbance, fewer parasite species infecting mammals, birds, and frogs as definitive hosts were found at both $\mathrm{Ag} 1$ and $\mathrm{AgP} 3$. This lends support to our second hypothesis. Moreover, the CCA attributed individual parasite species abundance at all localities to landscape variables associated with the wetlands, notably agricultural area and also urban area. The above results strongly suggest that definitive host movement to agricultural wetlands may be restricted by landscape development reducing habitat suitability and accessibility. In contrast, component and infracommunity species richness were consistently and relatively high at our reference localities, but not necessarily higher than at some of the perturbed localities. Thus, our first hypothesis may apply only when comparing reference localities with those more severely affected by environmental disturbance.

If modification of the landscape reduces the diversity of potential intermediate and definitive hosts present in the habitat, this should be accompanied by a reduction in parasite diversity (Hudson et al. 1998). Therefore, agricultural or urban expansion which can fragment and effectively reduce habitat visitation by birds and small mammals, may consequently affect parasite transmission from these hosts (Kuris and Lafferty, 1994; Keas and Blankespoor, 1997; Laffery, 1997; Smith, 2001; Huspeni and Lafferty, 2004; Lafferty and Kuris, 2005 ; Bradley and Altizer, 2006; Koprivnikar et al. 2006 a). This is supported by the rarity or complete absence of many parasite species from $\mathrm{Ag} 1$ and $\mathrm{AgP} 3$ that may use frogs as intermediate hosts and ultimately infect birds (e.g., Apharyngostrigea pipientis, Clinostomum sp., and Diplostomum spp.) and small mammals (e.g., Fibricola sp. in the case of Ag1 only, and Alaria sp. at both localities). In contrast to Ag1 and AgP3, other wetlands on both the $100 \mathrm{~m}$ and $500 \mathrm{~m}$ scale were typically surrounded by vegetation (e.g., forest) or were part of more extensive wetlands, providing the travel corridors and foraging habitat necessary for a greater abundance and variety of bird species (Shirley and Smith, 2005). Fragmentation of these habitats may cause a significant decline in visitation even by those waterbirds that do frequent agricultural wetlands, including herons (Ardea spp.) (Czech and Parsons, 2002), definitive hosts for Apharyngostrigea pipientis and Clinostomum sp. The distribution of small mammals may also be affected particularly if there is little to no buffer area surrounding aquatic habitats (Jones et al. 1988). Small mammals act as definitive hosts for many of the parasite species found in this study, including Alaria sp., Fibricola sp., and the echinostomes. In fact, Koprivnikar et al. (2006a) found that the prevalence of Alaria sp. in the gray tree frog (Hyla versicolor) was positively associated with forest cover, and therefore suggested that the forested landscapes provide potential canid hosts with greater access to the wetlands.

Similarly, loss of physical cover around the wetlands may have reduced the diversity of invertebrate intermediate hosts (Davies and Nelson, 1994; Jones et al. 1999; Environment Canada, 2005) at Ag1 and AgP3. While some studies have shown that agricultural activity can increase intermediate host abundance and consequently parasite transmission (Johnson and Chase, 2004; Beasley et al. 2005; McKenzie, 2007), this is not likely the case in this system. Reduced cover may explain the absence or low abundance of species acquired by frogs in their diet, such as Gorgoderina attenuata and Haematoloechus spp. Most adult trematodes of amphibians use aquatic or semi-aquatic arthropods or 
arthropod larvae as second intermediate hosts (Prudhoe and Bray, 1982), and development around freshwater lakes and streams can have significant negative effects on these invertebrate intermediate hosts. Indeed, low invertebrate diversity and abundance have been found in streams that are surrounded by land modified for agricultural and urban purposes (Beaven et al. 2001; Moore and Palmer, 2005). In heavily logged boreal watersheds, the low parasite infracommunity richness of northern redbelly dace (Phoxinus eos) was attributed to a reduction in the abundance of invertebrate intermediate hosts, particularly mayflies, as a result of the deforestation (Marcogliese et al. 2001). In contrast, Ref1 and AgP1 were the most forested on both $100 \mathrm{~m}$ and $500 \mathrm{~m}$ scales, and frogs from these wetlands were consistently infected with the greatest abundance of trophically transmitted parasites.

Reduced or impaired access to wetlands from habitat fragmentation and isolation can also hamper wetland habitat use by amphibians (Semlitsch and Bodie, 2003; Green, 2005) and, in fact, amphibians are often absent from intensely farmed areas (Loman and Lardner, 2006). Reduced amphibian diversity and abundance in agricultural wetlands would significantly limit opportunities for exchange of parasites among individual frogs, ultimately impeding the colonization of parasites requiring these animals as definitive hosts. In the present study, parasite species maintained by frog definitive hosts (Glypthelmins quieta, Gorgoderina attenuata, Haematoloechus spp., Cosmocercoides sp., Oswaldocruzia sp., Rhabdias ranae, and Strongyloides sp.) were rarely encountered, in low abundance, or absent at Ag1 and AgP3. Decreased forest cover and increased road density can negatively affect species richness in the amphibian community by impeding movement between wetlands, increasing the risk of predation and other mortality factors, and by making the habitat unsuitable for breeding (Houlahan and Findlay, 2003). The lower infection levels of the parasites listed above relative to those found at undeveloped or mildly developed wetlands may reflect reduced use of these wetland habitats by frogs.

Although many species encountered at reference and at other less disturbed agricultural wetlands were not present at Ag1 and AgP3, Echinostomatidae gen. sp. 1 was common at all localities. In fact, most frogs in both 2004 and 2005 were infected with the kidney echinostomes, a finding consistent with other studies of helminth parasites infecting eastern Canadian ranid populations (e.g., McAlpine and Burt, 1998; Koprivnikar et al. 2006a). This parasite is one of the most common and abundant in the otherwise impoverished communities at $\mathrm{Ag} 1$ and $\mathrm{AgP} 3$, and its abundance was positively associated with agricultural area and those two localities in the CCA. Other landscape perturbations, such as intense urbanization (Skelly et al. 2005) and loss of aquatic vegetation
(Beasley et al. 2005) have been linked with heavy echinostome infections in green frogs and cricket frogs, respectively. Heavy infections have been associated with adult leopard frog mortality and can be lethal to tadpoles of this species (Schotthoefer et al. 2003). The broad specificity of echinostomes may make it more likely that suitable hosts will exist in impacted environments. Indeed, species of echinostomes commonly use frogs as second intermediate hosts (Prudhoe and Bray, 1982; Kostadinova and Gibson, 2000), and infect a wide spectrum of hosts at different stages in their life-cycles. Their miracidia infect at least 4 genera of snails and cercariae infect most snails, fingernail clams, and tadpoles, while adults infect a variety of birds and mammals (McDonald, 1969; Olsen, 1974). The presence of highly host-specific parasites will theoretically decline when environmental disturbances cause host populations to be low, whereas generalist parasites and diseases should persist or even increase with environmental stress (Lafferty and Holt, 2003).

The parasite community and species abundance data suggest that intense landscape development from agriculture is more related to parasitism and the health of wetland ecosystems than pesticides, at least at the pesticide concentrations encountered in this study. Nevertheless, in addition to the landscape disturbances, high pesticide concentrations at AgP3 may have contributed to the markedly reduced parasitism observed here. Thus, this provides partial support for the third hypothesis that species richness should be reduced at localities affected by both landscape and pesticides. Although cluster analyses failed to differentiate among the 4 types of wetlands, it revealed $\mathrm{Ag} 1$ and $\mathrm{AgP} 3$ as the most dissimilar from others in terms of parasite occurrence, but also very dissimilar from each other. While both were surrounded by the most agricultural territory, Ag1 was nested within a managed community parkland and AgP3 had the highest pesticide levels of all localities. However, decreased parasitism in agricultural wetlands such as at AgP3 may also be due to direct toxicity of pesticides to free-living stages (Pietrock and Marcogliese, 2003). Koprivnikar et al. (2006b) found that $200 \mu \mathrm{g} / \mathrm{l}$ of atrazine affected the activity and survival of Alaria sp. cercariae and $20 \mu \mathrm{g} / 1$ atrazine reduced the infection success of Echinostoma sp. in Rana clamitans tadpoles. The results in the present study were somewhat consistent with their conclusions. Alaria sp. was absent at $\mathrm{AgP} 3$ and in $\mathrm{P} 2$ in 2004, and also rare in other agricultural wetlands, while infection levels of echinostomes at AgP3 in 2005 were among the lowest. Further investigations into how specific pesticides and pesticide mixtures affect the free-living stages of other parasite species known to infect amphibians are needed, particularly at concentrations more applicable to realistic field situations. 
The abundance of monoxenous nematodes was consistently lower in frogs from AgP3, and Rhabdias ranae was significantly more abundant in 2004 in non-contaminated wetlands than agricultural wetlands where pesticides had been detected. These results are contrary to reports from laboratory studies which demonstrated that exposure to aquatic pollution leads to increased establishment of directly transmitted parasites possibly as a result of immunosuppression (for fish-monogenean examples, see Khan and Thulin, 1991 and MacKenzie et al. 1995).Yet, immunosuppression due to pesticide exposure may still be a factor influencing trematode parasitism in the present study. Fibricola sp., which infects frogs by cutaneous penetration, was more abundant in contaminated agricultural localities in both years. This parasite species, along with Echinostomatidae gen. sp. 1, one of the most abundant parasite species in $\mathrm{AgP} 3$, infects frogs at the tadpole stage when the immune system is most susceptible to suppression from pesticide exposure (Carey and Bryant, 1995; Gilbertson et al. 2003). Thus, even though species richness was reduced at this locality, certain parasites were encountered in relatively high numbers, as predicted by our third hypothesis. Trematodes with a similar transmission mode, specifically Ribeiroia ondatrae and Telorchis sp., have been shown to increase in prevalence in immunosuppressed tadpoles exposed in the field to agricultural run-off containing pesticides (Kiesecker, 2002). Nevertheless, the relationship of landscape to parasitism is so strong in the current system that any additional patterns caused by pesticide effects may be obscured.

At times, the concentrations of atrazine at $\mathrm{AgP} 3$ were 10 to 100 times greater than those measured at the 4 other pesticide contaminated agricultural wetlands and exceeded the Canadian Water Quality Guildelines (2001) of $3 \mu \mathrm{g} / \mathrm{l}$ of atrazine for the protection of freshwater life. Parasite infracommunity species richness and diversity of frogs at moderately contaminated localities tended to be comparatively lower than those from reference localities, but not as low as those from $\mathrm{Ag} 1$ and $\mathrm{AgP} 3$. Thus, it appears that even moderate levels of pesticide contamination may affect parasite communities, although no significant trends were detected. The responses of parasite communities may be subtle or absent if a disturbance or pollution level is moderate (Marcogliese et al. 2006) and would be difficult to detect in part because the responses of all parasite species are integrated together in community analyses (Kennedy, 1997; Lafferty, 1997; Marcogliese, 2005). On the other hand, parasite communities may only respond noticeably if the disturbance exceeds a certain magnitude or threshold. This would also explain why we only have equivocal support for our first hypothesis, that parasite species richness would be greatest in localities free from agricultural influence.
Variation in certain water quality parameters, other than pesticides, was related to parasite species richness and abundance. Conductivity and dissolved organic carbon were significant predictors of component community composition. In fact, dissolved organic carbon was also positively associated with component community species richness and the abundance of most parasite species. This suggests that dissolved organic carbon reflects environmental conditions that sustain a highly diversified parasite community. Dissolved organic carbon can mediate the density of invertebrates in freshwater systems through trophic pathways (Wetzel, 2001). High concentrations of dissolved organic carbon can cause shifts in the invertebrate community structure from small invertebrate species to large predatory invertebrates and insect larvae (Wissel et al. 2003). This could affect the transmission of parasite species such as Gorgoderina attenuata, Haematoloechus spp., and Spiroxys sp. that use insect larvae and other large aquatic invertebrates as second intermediate hosts. Interestingly, conductivity was strongly and positively associated with the abundance of gorgoderids (both adult and metacercariae) in the CCA. Conductivity generally reflects the ion content of water. Waters of low conductivity that are low in biologically important ions may not be suitable habitats for sphaeriid clams (McMahon and Bogan, 2001), the first intermediate hosts of gorgoderid parasites. Differences in density of sphaeriid clam populations among the localities may explain the association of gogorgoderid trematodes with conductivity. These differences may not have been detected in our limited invertebrate sampling regime, which also was compromised by intense rainfall during the sampling period.

In conclusion, our results generally supported the hypotheses of the study. Firstly, frogs inhabiting reference localities were infected by the greatest number of parasite species at the component and infracommunity level. Secondly, frogs from localities surrounded by large agricultural landscapes were infected with fewer species that use birds and mammals as definitive hosts and fewer species overall. This was largely attributed to fewer definitive hosts using the wetland habitats, consequently reducing the transmission of their parasites to the metamorph leopard frogs in those habitats. Thirdly, the most perturbed locality with both the highest pesticide levels and the greatest area of agricultural landscape had, along with a partially urbanized and agricultural locality, frogs infected with the fewest parasite species. While frogs from anthropogenically disturbed wetlands may be infected with fewer parasite species, this does not suggest that frogs in these environments are indeed in better health, for there may be deleterious interactions between contaminants and parasites (e.g., Marcogliese et al. 2005). Furthermore, the fact that depauperate 
parasite communities likely reflect impoverished host communities suggests that these wetlands are seriously impacted and that the parasite communities of frogs appear to be good indicator organisms of ecosystem health. These observations should be considered in conservation planning in response to amphibian declines.

We thank Sean Locke, Lila Brambilla-Gagnon, Sophie Trépanier, Sonia Roy, Jean-Martin Chamberland, Michel Arseneau, Germain Brault, Claude Lessard, and Katia Étienne from the St Lawrence Centre (Environment Canada) for assistance in the collections. Denis Laliberté, Christian DeBlois, and Nathalie Dassylva contributed to the nutrient and pesticide analysis. Pierre Gagnon kindly provided statistical advice. François Boudreault generously prepared the map and produced the landscape data, and the voucher parasite specimens provided by Judith Price and Donald McAlpine greatly helped in our identifications. Thanks also go to John Sauro and Raymond Greffe who permitted us to sample on their lands, along with Danielle Chatillon of the Société des établissements de plein-air du Québec who facilitated access to Île de la Commune wetland. We are also grateful to Valerie McKenzie for her helpful comments. Funding for this work was provided by the Pesticide Science Fund (Environment Canada), an NSERC Postgraduate Scholarship awarded to K.C.K., and an NSERC Discovery Grant to J.D.M.

\section{REFERENCES}

Amezaga, J. M., Santamaría, L. and Green, A. J. (2002). Biotic wetland connectivity - supporting a new approach for wetland policy. Acta Oecologica 23, 213-222.

Beasley, V. R., Faeh, S. A., Wikoff, B., Staehle, C., Eisold, J., Nichols, D., Cole, R., Schotthoefer, A. M., Greenwell, M. and Brown, L. E. (2005). Risk factors and declines in northern cricket frogs (Acris crepitans). In Declining Amphibians : a United States Response to the Global Phenomenon (ed. Lannoo, M. J.), pp. 75-86. University of California Press, Berkeley, USA.

Beaven, L., Sadler, J. and Pinder, C. (2001). The invertebrate fauna of a physically modified urban river. Hydrobiologia 445, 97-108.

Bradley, C. A. and Altizer, S. (2006). Urbanization and the ecology of wildlife diseases. Trends in Ecology and Evolution 22, 95-102.

Bush, A. O., Lafferty, K. D., Lotz, J. M. and Shostak, A. W. (1997). Parasitology meets ecology on its own terms: Margolis et al. revisited. Fournal of Parasitology 83, 575-583.

Canadian Council of Ministers of the Environment. (2001). Canadian Water Quality Guidelines (CWQG) for the Protection of Aquatic Life. Environment Canada, Ottawa.

Carey, C., Bradford, D. F., Brunner, J. L., Collins, J. P., Davidson, E. W., Longcore, J. E., Ouellet, M., Pessier, A. P. and Schock, D. M. (2003). Biotic factors in amphibian population declines. In Amphibian Decline: An Integrated Analysis of Multiple Stressor Effects (ed. Linder, G. L., Krest, S. and Sparling, D.), pp. 153-208. Society of Environmental Toxicology and Chemistry (SETAC), Pensacola, FL, USA.
Carey, C. and Bryant, C. J. (1995). Possible interrelations among environmental toxicants, amphibian development, and decline of amphibian populations. Environmental Health Perspectives 103 (Suppl.), S13-S17.

Carpenter, S. R., Caraco, N. F., Correll, D. L., Howarth, R. W., Sharpley, A. N. and Smith, V. H. (1998). Non-point pollution of surface waters with phosphorus and nitrogen. Ecological Applications 8, 559-568.

Carrier, S. (2001). National Laboratory for Environmental Testing, 2001-02 Sample Collection Summary. Environment Canada, National Water Research Institute, Burlington/Saskatoon.

Christin, M. S., Gendron, A. D., Brousseau, P., Menard, L., Marcogliese, D. J., Cyr, D., Ruby, S. and Fournier, M. (2003). Effects of agricultural pesticides on the immune system of Rana pipiens and on its resistance to parasitic infection. Environmental Toxicology and Chemistry 22, 1127-1133.

Christin, M. S., Ménard, L., Gendron, A. D., Ruby, S., Cyr, D., Marcogliese, D. J., Rollins-Smith, L. and Fournier, M. (2004). Effects of agricultural pesticides on the immune system of Xenopus laevis and Rana pipiens. Aquatic Toxicology 67, 33-43.

Clarke, A. H. (1981). The Freshwater Molluscs of Canada. National Museums of Canada, Ottawa.

Cone, D. K., Marcogliese, D. J. and Watt, W. D. (1993). Metazoan parasite communities of yellow eels (Anguilla rostrata) in acidic and limed rivers of Nova Scotia. Canadian Fournal of Zoology 71, 177-184.

Conover, W. J. and Iman, R. L. (1981). Rank transformations as a bridge between parametric and nonparametric statistics. American Statistician 35, 124-136.

Czech, H. A. and Parsons, K. C. (2002). Agricultural wetlands and waterbirds: a review. Waterbirds 25 (Special Publication 2), 25-41.

Daszak, P., Cunningham, A. A. and Hyatt, A. D. (2003). Infectious disease and amphibian population declines. Diversity and Distributions 9, 141-150.

Davies, P. E. and Nelson, M. (1994). Relationships between riparian buffer widths and the effects of logging on stream habitat, invertebrate community composition and fish abundance. Australian Fournal of Marine and Freshwater Research 45, 1289-1305.

Desroches, J. F. and Rodrigue, D. (2004). Amphibiens et reptiles du Québec et des maritimes. Éditions Michel Quintin, Waterloo.

Gendron, A. D., Marcogliese, D. J., Barbeau, S., Christin, M. S., Brousseau, P., Ruby, S., Cyr, D. and Fournier, M. (2003). Exposure of leopard frogs to a pesticide mixture affects life history characteristics of the lungworm Rhabdias ranae. Oecologia 135, 469-476.

Gibbs, J. P. (2000). Wetland loss and biodiversity conservation. Conservation Biology 14, 314-317.

Gilbertson, M. K., Haffner, G. D., Drouillard, K. G., Albert, A. and Dixon, B. (2003). Immunosuppression in the northern leopard frog (Rana pipiens) induced by pesticide exposure. Environmental Toxicology and Chemistry 22, 101-110.

Gillilland III, M. G. and Muzzall, P. M. (1999). Helminths infecting froglets of the northern leopard frog (Rana pipiens) from Foggy Bottom Marsh, Michigan. 
Fournal of the Helminthological Society of Washington 66, 73-77.

Giroux, I. (1999). Contamination de l'eau par les Pesticides dans les Régions de Culture de Maïs et de Soya au Québec. Direction des écosystèmes aquatiques, Ministère de l'Environnement, Quebec.

Giroux, I. (2002). Contamination de l'eau par les Pesticides dans les Régions de Culture de Maï et de Soya au Québec. Direction des écosystèmes aquatiques, Ministère de l'Environnement, Quebec.

Goater, T. M. and Goater, C. P. (2001). Ecological monitoring and assessment network: Protocols for measuring biodiversity: Parasites of amphibians and reptiles. http://www.eman-rese.ca/eman/ecotools/ protocols/terrestrial/herp_parasites/intro.html

Green, D. M. (2005). The biology of amphibian declines. In Declining Amphibians : a United States Response to the Global Phenomenon (ed. Lannoo, M. J.), pp. 58-72. University of California Press, Berkeley, USA.

Hamann, M. I., Kehr, A. I. and González, C. E. (2006). Species affinity and infracommunity ordination of helminths of Leptodactylus chaqensis (Anura: Letpdactylidae) in two contrasting environments from northeastern Argentina. Fournal of Parasitology 92, 1171-1179.

Hechinger, R. F. and Lafferty, K. D. (2005). Host diversity begets parasite diversity: bird final hosts and trematodes in snail intermediate hosts. Proceedings of the Royal Society of London, B 272, 1059-1066.

Hernandez, A. D., Bunnel, J. F. and Sukhdeo, M. V. K. (2007). Composition and diversity patterns in metazoan parasite communities and anthropogenic disturbance in stream ecosystems. Parasitology $\mathbf{1 3 4}$, 91-102.

Heyer, R. A., Donnelly, M. A., McDiarmid, R. W., Hayek, L.-A. C. and Foster, M. S. (1994). Measuring and Monitoring Biological Diversity: Standard Methods for Amphibians. Smithsonian Institution Press, Washington.

Houlahan, J. E. and Findlay, C. S. (2003). The effects of adjacent land use on wetland amphibian species richness and community composition. Canadian Fournal of Fisheries and Aquatic Sciences 60, 1078-1094.

Hudson, P. J., Dobson, A. P. and Lafferty, K. D. (2006). Is a healthy ecosystem one that is rich in parasites? Trends in Ecology and Evolution 7, 381-385.

Hudson, P. J., Dobson, A. P. and Newborn, D. (1998). Prevention of population cycles by parasite removal. Science 282, 2256-2258.

Huspeni, T. C. and Lafferty, K. D. (2004). Using larval trematodes that parasitize snails to evaluate a saltmarsh restoration project. Ecological Applications 14, 795-804.

Johnson, P. T. J. and Chase, J. M. (2004). Parasites in the food web: linking amphibian malformations and aquatic eutrophication. Ecology Letters 7, 521-526.

Jones, J. J., Lortie, J. P. and Pierce, U. D. (1988). The Identification and Management of Significant Fish and Wildlife Resources in Southern Coastal Maine. Maine Department of Inland Fisheries and Wildlife, Augusta, Maine, USA.

Jones, E. B. D., Helfman, G. S., Harper, J. O. and Bolstad, P. V. (1999). Effects of riparian forest removal on fish assemblages in southern Appalachian streams. Conservation Biology 13, 1454-1465.
Keas, B. and Blankespoor, H. D. (1997). The prevalence of cercariae from Stagnicola emarginata over 50 years in Northern Michigan. Fournal of Parasitology 83, 536-540

Kennedy, C. R. (1997). Freshwater fish parasites and environmental quality: an overview and caution. Parassitologia 39, 249-254.

Khan, R. A. and Thulin, J. (1991). Influence of pollution on parasites of aquatic animals. Advances in Parasitology 30, 201-238.

Kiesecker, J. M. (2002). Synergism between trematode infection and pesticide exposure: a link to amphibian limb deformities in nature? Proceedings of the National Academy of Sciences, USA 99, 9900-9904.

Kiesecker, J. M., Belden, L. K., Katriona, S. and Rubbo, M. J. (2004). Amphibian decline and emerging disease: What can sick frogs teach us about new and resurgent diseases in human populations and other species of wildlife? American Scientist 92, 139-147.

Kiesecker, J. M., Blaustein, A. R. and Belden, L. K. (2001). Complex causes of amphibian population declines. Nature, London 410, 681-684.

Koprivnikar, J., Forbes, M. R. and Baker, R. L. (2006a). Environmental factors influencing trematode prevalence in the grey tree frog (Hyla versicolor) tadpoles in southern Ontario. Fournal of Parasitology 92, 997-1001.

Koprivnikar, J., Forbes, M. R. and Baker, R. L. $(2006 b)$. Effects of atrazine on cercarial longevity, activity, and infectivity. Fournal of Parasitology 92, 306-311.

Kostadinova, A. and Gibson, D. I. (2000). The systematics of the echinostomes. In Echinostomes as Experimental Models for Biological Research (ed. Fried, B. and Graczyk, T. K.), pp. 31-57. Kluwer, Dordrecht, The Netherlands.

Kuris, A. M. and Lafferty, K. D. (1994). Community structure: larval trematodes in snail hosts. Annual Review of Ecology and Systematics 25, 189-217.

Laboratoire du Centre Saint-Laurent. (1994). Manuel des Méthodes d'Analyse: Partie 1 (Analyses PhysicoChimiques). Partie 2 (Analyse des Paramètres Inorganiques). Environnement Canada, Conservation de l'environnement, Centre Saint-Laurent, Montreal.

Lafferty, K. D. (1997). Environmental parasitology: what can parasites tell us about human impacts on the environment? Parasitology Today 13, 251-255.

Lafferty, K. D. and Holt, R. D. (2003). How should environmental stress affect the population dynamics of disease? Ecology Letters 6, 654-664.

Lafferty, K. D. and Kuris, A. M. (1999). How environmental stress affects the impacts of parasites. Limnology and Oceanography 44, 925-931.

Lafferty, K. D. and Kuris, A. M. (2005). Parasitism and environmental disturbances. In Parasitism and Ecosystems (ed. Thomas, F, Guégan, J. F. and Renaud, F.), pp. 113-123. Oxford University Press, London.

Laurila, A., Pakkasmaa, S., Crochet, P. A. and Merilä, J. (2002). Predator-induced plasticity in early life-history and morphology in two anuran amphibians. Oecologia 132, 524-530.

Laurila, A., Pakkasmaa, S. and Merilä, J. (2001). Influence of seasonal time constraints on growth and 
development of common frog tadpoles: a photoperiod experiment. Oikos 95, 451-460.

Leclair, R. and Castanet, J. (1987). Skeletochronological assessment of age and growth in the frog Rana pipiens Schreber (Amphibia, Anura) from Southwestern Quebec. Copeia 2, 361-369.

Legendre, P. and Legendre, L. (1998). Numerical Ecology, 2nd Edn. Elsevier, Oxford.

Lemieux, C., Quémerais, B. and Lum, K. R. (1995). Seasonal patterns of atrazine loading for the St. Lawrence River (Canada) and its tributaries. Water Research 29, 1491-1504.

Loman, J. and Lardner, B. (2006). Does pond quality limit frogs Rana arvalis and Rana temporaria in agricultural landscapes? A field experiment. Fournal of Applied Ecology 43, 690-700.

MacKenzie, K., Williams, H. H., Williams, B., McVicar, A. H. and Siddall, R. (1995). Parasites as indicators of water quality and the potential use of helminth transmission in marine pollution studies. Advances in Parasitology 35, 85-114.

MacKenzie, K. (1999). Parasites as pollution indicators in marine ecosystems : a proposed early warning system. Marine Pollution Bulletin 38, 955-959.

Magurran, A. E. (1988). Ecological Diversity and Its Measurement. Princeton University Press, Princeton, USA.

Marcogliese, D. J. (2003). Food webs and biodiversity: are parasites the missing link? Fournal of Parasitology (Suppl.) 89, S106-S113.

Marcogliese, D. J. (2004). Parasites: small players with crucial roles in the ecological theatre. Ecohealth 1, 151-164.

Marcogliese, D. J. (2005). Parasites of the superorganism: are they indicators of ecosystem health? International Fournal for Parasitology 35, 705-716.

Marcogliese, D. J., Ball, M. and Lankester, M. W. (2001). Potential impacts of clearcutting on parasites of minnows in small boreal lakes. Folia Parasitologica 48, 269-274.

Marcogliese, D. J. and Cone, D. K. (1997). Parasite communities as indicators of ecosystem stress. Parassitologia 39, 227-232.

Marcogliese, D. J., Gagnon Brambilla, L., Gagné, F. and Gendron, A. D. (2005). Joint effects of parasitism and pollution on biomarkers of oxidative stress in yellow perch (Perca flavescens). Diseases of Aquatic Organisms 63, 77-84.

Marcogliese, D. J., Gendron, A. D., Plante, C., Fournier, M. and Cyr, D. (2006). Parasites of spottail shiners (Notropis hudsonius) in the St. Lawrence River: effects of municipal effluents and habitat. Canadian Fournal of Zoology 84, 1461-1481.

Matson, P. A., Parton, W. J., Power, A. G. and Swift, M. J. (1997). Agricultural intensification and ecosystem properties. Science 277, 504-509.

McAlpine, D. F. and Burt, M. D. B. (1998). Helminths of bullfrogs (Rana catesbeiana), green frogs (Rana clamitans), and leopard frogs (Rana pipiens) in New Brunswick. Canadian Field-Naturalist 112, 50-68.

McDonald, M. E. (1969). Annotated Bibliography of Helminths of Waterfowl (Anatidae). Bureau of Sport
Fisheries and Wildlife Special Scientific Report No. 125, Washington.

McKenzie, V. J. (2007). Human land use and patterns of parasitism in tropical amphibian hosts. Biological Conservation 137, 102-116.

McMahon, R. F. and Bogan, A. E. (2001). Mollusca: Bivalvia. In Ecology and Classification of North American Freshwater Invertebrates, 2nd Edn (ed. Thorp, J. H. and Covich, A. P.), pp. 297-325. Academic Press, San Diego, USA.

Moore, A. A. and Palmer, M. A. (2005). Invertebrate biodiversity in agricultural and urban headwater streams: implications for conservation and management. Ecological Applications 15, 1169-1177.

Muzzall, P. M. (2005). Parasites of Amphibians and Reptiles from Michigan: A Review of the Literature 1916-2003. Fisheries Research Report No. 2077. State of Michigan Department of Natural Resources, Ann Arbor, USA.

Environment Canada. (2005). A Literature Review of Risks and Benefits of Agriculture to Biodiversity and Biodiversity to Agriculture. National AgriEnvironmental Standards Initiative Technical Series Report 1-15, Gatineau, Quebec, Canada.

Olsen, O. W. (1974). Animal Parasites: Their Life Cycles and Ecology, 3rd Edn. University Park Press, Baltimore, USA.

Overstreet, R. M. and Howse, H. D. (1977). Some parasites and diseases of estuarine fishes in polluted habitats of Mississippi. Annals of the New York Academy of Sciences 298, 437-462.

Pietrock, M. and Marcogliese, D. J. (2003). Free-living endohelminth stages: at the mercy of environmental conditions. Trends in Parasitology 19, 293-298.

Poulin, R. (1992). Toxic pollution and parasitism in freshwater fish. Parasitology Today 8, 58-61.

Prudhoe, S. and Bray, R. A. (1982). Platyhelminth Parasites of the Amphibia. Oxford University Press, London.

Rau, M. E., Doyle, J. and Gordon, D. (1978). Les parasites des animaux sauvages du Québec. 2. Les parasites des grenouilles et des serpents de la région de l'île Perrot. Le Naturaliste Canadien 105, 56-57.

Relyea, R. A. (2004). Growth and survival of five amphibian species exposed to combinations of pesticides. Environmental Toxicology and Chemistry 23, 1737-1742.

Schotthoefer, A. M., Cole, R. A. and Beasley, V. R. (2003). Relationship of tadpole stage to location of echinostome cercariae encystment and the consequences for tadpole survival. Fournal of Parasitology 89, 475-482.

Scott, D. E. (1990). Effect of larval density of Ambystoma opacum: an experiment in large-scale field enclosures. Ecology 71, 296-306.

Seburn, C. N. L. and Seburn, D. C. (1998). Status Report: Northern Leopard Frog Rana pipiens (Western Population). Committee on the Status of Endangered Wildlife in Canada, Ottawa.

Semlitsch, R. D. and Bodie, J. R. (2003). Biological criteria for buffer zones around wetlands and riparian habitats for amphibians and reptiles. Conservation Biology 17, 1219-1228. 
Semlitsch, R. D., Scott, D. E. and Pechmann, J. H. K. (1988). Time and size at metamorphosis related to adult fitness in Ambystoma talpoideum. Ecology 69, 184-192.

Shirley, S. M. and Smith, J. N. M. (2005). Bird community structure across riparian buffer strips of varying width in a coastal temperature forest. Biological Conservation 125, 475-489.

Skelly, D. K., Bolden, S. R., Holland, M. P., Freidenburg, L. K., Freidenfelds, N. A. and Malcolm, T. R. (2005). Urbanization and disease in amphibians. In Disease Ecology: Community Structure and Pathogen Dynamics (ed. Collinge, S. K. and Ray, C.), pp. 153-167. Oxford University Press, Oxford.
Smith, N. F. (2001). Spatial heterogeneity in recruitment of larval trematodes to snail intermediate hosts. Oecologia 127, 115-122.

Ter Braak, C. J. F. and Šmilauer, P. (1998). CANOCO Reference Manual and User's Guide to Canoco for Windows : Software for Canonical Community Ordination (Version 4.0). Centre for Biometry, Wageningen, The Netherlands.

Wetzel, R. G. (2001). Limnology. Saunders College Publishing, Philadelphia, USA.

Wissel, B., Boeing, W. J. and Ramcharan, C. W. (2003). Effects of water color on predation regimes and zooplankton assemblages in freshwater lakes. Limnology and Oceanography 48, 1965-1976. 